\title{
Effective Conveyor Belt Inspection for Improved Mining Productivity \\ Final Report
}

\author{
Version 1.0 \\ Reporting Period: November 15, 2004 - November 14, 2007 \\ Issued: March, 2008 \\ US Department of Energy \\ Award \#DE-FC26-04NT42086 \\ Principal Author: \\ DavidLaRose,dlr@cs.cmu.edu \\ National Robotics Engineering Center \\ Carnegie Mellon University \\ Ten $40^{\text {th }}$ Street \\ Pittsburgh, Pennsylvania 15201
}

\section{Technical Contact:}

David LaRose

Tel: 412-327-3970

Email: dlr@cs.cmu.edu
Administrative Contact:

Susan Burkett

Tel: 412-268-1975

Email: sburkett@andrew.cmu.edu

Document No. 1135600008 
This report was prepared as an account of work sponsored by an agency of the United States Government. Neither the United States Government nor any agency thereof, nor any of their employees, makes any warranty, express or implied, or assumes any legal liability or responsibility for the accuracy, completeness, or usefulness of any information, apparatus, product, or process disclosed, or represents that its use would not infringe privately owned rights. Reference herein to any specific commercial product, process, or service by trade name, trademark, manufacturer, or otherwise does not necessarily constitute or imply its endorsement, recommendation, or favoring by the United States Government or any agency thereof. The views and opinions of authors expressed herein do not necessarily state or reflect those of the United States Government or any agency thereof. 


\begin{abstract}
This document details progress on the project "Effective Conveyor Belt Inspection for Improved Mining Productivity," funded under U.S. Department of Energy award number DEFC26-04NT42086. The goal of the project was to develop automated conveyor belt inspection technology for use in underground underground and surface mines, coal-fired power generation plants, and other large scale material handling operations. Appropriate technologies have been developed and implemented, product prototypes are in daily use at 16 active mines, and commercialization efforts are well underway.
\end{abstract}




\section{Contents}

1 Introduction 6

2 Executive Summary 6

3 Experimental $\quad 8$

3.1 Mechanical Splice Detection System . . . . . . . . . . . . . . . . 8

3.1 .1 Test Facility . . . . . . . . . . . . . . . . . . 8

3.1.2 Design of PC-Based BeltVision Prototype . . . . . . . . . . . . . . . 9

3.1 .3 Lighting Redesign . . . . . . . . . . . . . . . . . . . . . . 11

3.2 Smart Camera Development . . . . . . . . . . . . . . . . . . 12

3.2.1 Completion of Laboratory Prototype . . . . . . . . . . . . . . . . . . . . . . . . . . . . . . . . .

3.2.2 Final Design and System Implementation. . . . . . . . . . . . . . . . . . 14

3.2.3 Embedded Software Development . . . . . . . . . . . . . . . . . . . 15

3.3 Infrared Detection of Vulcanized Splices . . . . . . . . . . . . . . . . . 16

3.3.1 Experimental Apparatus . . . . . . . . . . . . . . . . . . 17

3.4 Vision-Based Detection of Vulcanized Splices . . . . . . . . . . . . . . . . 17

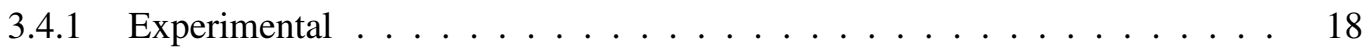

3.5 Lighting Refinements . . . . . . . . . . . . . . . . . . . . . . 18

4 Results and Discussion $\quad 19$

4.1 Mechanical Splice Detection System . . . . . . . . . . . . . . . . . . . . 19

4.2 Smart Camera Development . . . . . . . . . . . . . . . . . . . . . . . . . 19

4.3 Infrared Detection of Vulcanized Splices . . . . . . . . . . . . . . . . . . 21

4.4 Vision-Based Detection of Vulcanized Splices . . . . . . . . . . . . . . . . . 21

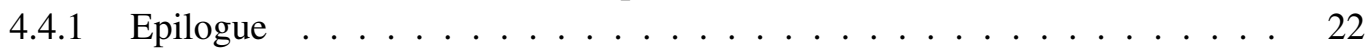

5 Conclusion 29 


\section{List of Figures}

1 A full-sized test rig has been built and installed at NREC for testing and algorithm development. . . . . . . . . . . . . . . . . 9

2 Original belt inspection prototype system installed in an underground coal mine. . .

3 A reduced cost BeltVision prototype was designed in order to facilitate testing, marketing, and deployment. The new design is highly modular, permitting easy replacement of parts. Subfigure (a) shows inexpensive, off-the-shelf computing hardware in a protective enclosure, and Subfigure (b) shows a fluorescent lightbar assembly. Note that the fluorescent light bar was subsequently replaced by an LED based unit.

4 A schematic of the PC-based BeltVision system. For photos of the actual system, please refer to Figure $5 \ldots \ldots \ldots \ldots \ldots$

5 The PC-based BeltVision system includes two lightbars which illuminate the belt as it passes underneath the system. A pair of line-scan cameras acquire images of the belt, while processing of the acquired image data is performed in a nearby enclosure containing a commercial PC computer. . . . . . . . . . . . . . . .

6 Individual scanlines are assembled into $2 \mathrm{D}$ images, which are saved to disk. . . . .

7 Several different line-scan sensing elements were evaluated: (a) shows an image from a Dalsa CCD-based line-scan element; (b) shows a similar image from a Perkin-Elmer CCD-based line-scan element which is significantly less expensive; and (c) shows an image from a CMOS line-scan element, which was even less expensive, but which was found to have inadequate image quality. . . . . . . . . .

8 Overview of the initial low-cost Smart Camera system design. This illustration shows the sensor element integrated with the camera housing. In the final system, the sensor (a line-scan CCD array) is housed separately in a remotely attached sensor head, so that multiple CCDs can be driven by a single Smart Camera. . . . . . .

9 A schematic showing the mounting position of the Smart Camera prototype during the proof of concept demonstration. . . . . . . . . . . . . . . . 14

10 A partially assembled camera head for the Smart Camera based BeltVision system. 15

11 A fully functional computing board for the Smart Camera, photographed during demonstration in September, 2006. . . . . . . . . . . . . . . 16

12 A bench test of a single-headed Smart Camera system was conducted in late 2006.

13 The vulcanized splice detector allows incremental processing of image data. In the current installations, direct connection between the cameras and the vulcanized splice detection software is not implemented. Instead, splice detection is automatically performed twice a day using images which have been saved to hard disk. as

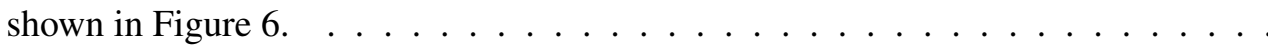

14 A screenshot of the GUI-based vulcanized splice detection application under development. Note that the inclusion of white fiducial markings in the neighborhood of vulcanized splices is very rare: the white cross visible in the screenshot is highly unusual. . . . . . . . . . . . . . . . . . 
15 (a) The original lighting configuration, using two light bars, deployed at Beitzel Corporation's above-ground test facility. The photograph is taken from beside the belt, looking perpendicular to the direction of belt travel, along the length of the light bars. A yellow calibration target is lying across the width of the belt. (b) The revised, single-light-bar configuration deployed underground. The light bar is partially obscured by a piece of brattice cloth, with the LED light emitting surface facing the camera. The brattice cloth curtain significantly reduces fouling of the cameras and light bar. . . . . . . . . . . . . . . . . . .

16 (a) The two-headed Smart Camera under test at the above-ground test facility. A conveyor belt passes underneath two LED light bars. The two yellow circles indicate the new camera heads, which are considerably smaller and less expensive than the Dalsa units that were previously used. (b) An LCD screen being driven by the Smart Camera. The top of the screen shows an image of the most recently detected mechanical splice, while the bottom of the screen shows an GUI overlay that can be manipulated via touchscreen. . . . . . . . . . . . . . . . . .

17 (a) The Smart Camera main board installed in wall-mounted enclosure in the Bailey mine for testing. The transparent cover of the enclosure folds down to allow access to an LCD touchscreen. The cover can be closed to protect the touchscreen without obscuring the display. (b) The light bar and two camera heads are suspended above the belt on an aluminum frame. This picture was taken on an operating belt, and the assembly is partially obscured by brattice cloth, which greatly reduces fouling of the cameras and light bar cover. . . . . . . . . . . . . . . . .

18 The thermographic imaging interface during acquisition of footage from the Blacksville mine. . . . . . . . . . . . . . . . . 22

19 Raw images from each of the four tested installation sites. . . . . . . . . . . 23

20 Sections of the images from the Bailey Mine and Robinson Run Mine. The contrast of these images has been increased somewhat to permit easy viewing. Pulley lagging in the Bailey mine image leaves an impression on the belt which is easily mistaken for the diagonal line of a vulcanized splice. . . . . . . . . . . . . . . 24

21 Representative splice images from each of the four tested installation sites. . . . . 25

22 These are the same images a shown in Figure 21, except that the contrast of each image has been adjusted to make the splice more easily visible. The Bailey Mine image is not visibly changed because the original image has very high contrast. . . 26

23 ROC curves for the Bailey and Robinson Run Mine datasets. . . . . . . . . . . . .

24 Belt images showing the effect of the new alignment algorithm. Contrast has been altered to make splice features more visible. (a) An image of a vulcanized splice from the from the Bailey mine without alignment. (b) The same image after alignment. (c) An image of a vulcanized splice from the Robinson Run mine without alignment. (d) The same image after alignment. . . . . . . . . . . . . . . . . 


\section{Introduction}

This report presents technical progress on the Effective Conveyor Belt Inspection for Improved Mining Productivity project, funded under U.S. Department of Energy award number DE-FC2604 NT42086.

The overall objective of the three-year project is to develop and commercialize a low cost conveyor belt inspection system for use in underground and surface mines, coal-fired power generation plants, and other large scale material handling operations. Mining operations rely on conveyor belts to move mined material from the working face to a processing plant. When a conveyor belt breaks or its operation is halted for unscheduled maintenance, there are often high costs in terms of lost productivity decreased mine efficiency, and wasted energy. For example, a mainline belt break in a longwall mining operation generally stops production for at least four hours. Concomitant revenue losses typically exceed $\$ 240,000$. CONSOL Energy has identified belt availability as one of the top two productivity bottlenecks in their operations today. Furthermore, belt repair under typical mine conditions is difficult, dangerous work.

If an effective belt inspection system is in place, belts that showed signs of degradation can be scheduled for repair during planned system downtime, eliminating negative impact on production while improving worker safety. Current belt inspection methods are ineffective. Manual inspection is time-consuming, misses many defects due to belt speed of 500-800 ft/min., and is sometimes very difficult due to insufficient belt access. Numerous belt condition monitoring technologies have been researched but very few have penetrated the mining market due to high costs, poor reliability, and high false positive rates. To address this need, we have developed a machine vision-based belt inspection system that helps operators identify belt splices that are close to failing.

The potential impact of this project on the overall mining industry is enormous. If a longwall mine producing 10 million tons per year were to install these systems for all of its conveyor belts, we estimate a productivity increase of $2 \%$ due to reduced belt downtime. This improvement produces $\$ 5,000,000$ (@\$25/ton) of additional revenue (if at full capacity) and reduces energy consumption per ten million tons produced by over 25 billion BTUs. This savings is due to the high energy cost of mine lighting, ventilation, and other operations during unproductive shifts, and is illustrated in Table 1. Initial market studies indicate that these savings would justify cost effective installations in nearly 7000 belts worldwide.

\section{Executive Summary}

At commencement of the project, a prototype belt inspection system had been developed under independent funding. This system was able to detect mechanical splices, but was not able to detect vulcanized splices. This restriction, along with the targeted selling price of approximately $\$ 75,000$, greatly limited the range of applications for which the prototype system was economically viable. The goal of this DOE-funded project was to address these limitations through several steps:

- Expand the capability of the belt inspection system to detect vulcanized splices.

- Increase system robustness to be capable of surviving the mining environment.

- Decrease targeted system selling price to less than $\$ 25,000$. 


\begin{tabular}{|c|c|c|c|c|c|}
\hline \multicolumn{6}{|c|}{ ENERGYBENEFTSTABLE } \\
\hline ENERGY SOURCE & $\begin{array}{l}\text { CURRENT } \\
\text { TECHNOLOGY } \\
\text { (BTU/NR/UNT) }\end{array}$ & $\begin{array}{l}\text { PROPOSED } \\
\text { TECHNOLOGY } \\
\text { (BTU/YR/UNIT) }\end{array}$ & $\begin{array}{c}\text { ENERGY SAVINGS } \\
\text { (BTU/YR/UNIT) }\end{array}$ & $\begin{array}{l}\text { NO. OF UNITS IN } \\
\text { 10 YEARS }\end{array}$ & $\begin{array}{c}\text { CUMULATIVE } \\
\text { ENERGY SAVINGS } \\
\text { (BTU/YR/UNT) }\end{array}$ \\
\hline & (A) & (B) & $(C=A B)$ & (D) & (E) \\
\hline \multicolumn{6}{|l|}{$\begin{array}{l}\text { OIL/GASOLINE/ } \\
\text { DIESEL }\end{array}$} \\
\hline \multicolumn{6}{|l|}{ NATURAL GAS } \\
\hline \multicolumn{6}{|l|}{ COAL } \\
\hline ELECTRICITY + & $964.2 \times 10^{9}$ & $945.0 \times 10^{9}$ & $19.2 \times 10^{9}$ & 50 & $960.0 \times 10^{9}$ \\
\hline \multicolumn{6}{|l|}{ OTHER ENERGY } \\
\hline TOTAL PER UNIT & $964.2 \times 10^{9}$ & $945.0 \times 10^{9}$ & $19.2 \times 10^{9}$ & 50 & $960.0 \times 10^{9}$ \\
\hline \multicolumn{6}{|c|}{ 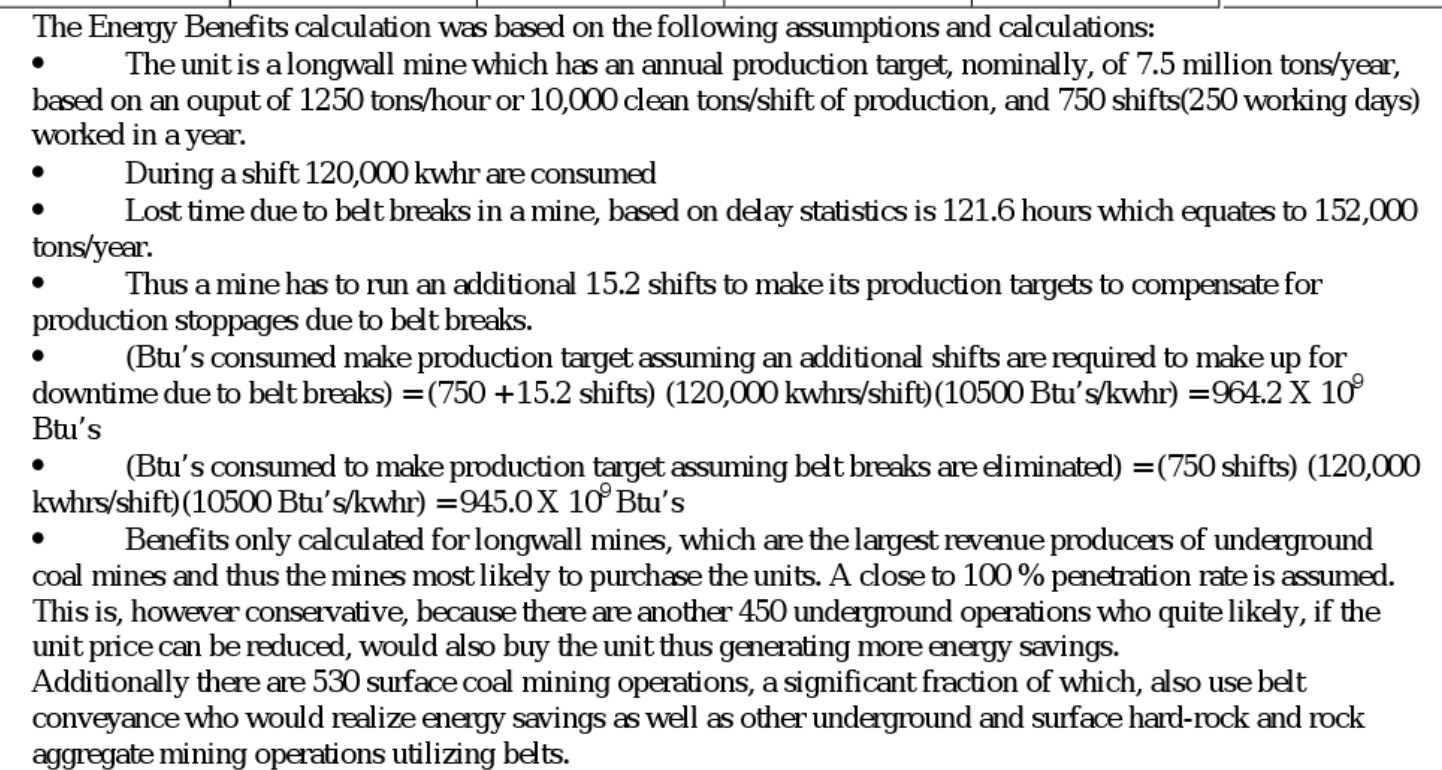 } \\
\hline
\end{tabular}

Table 1: Potential energy benefits of the developed system. 
- If possible, integrate additional functionality, such as rip detection, assessment of edge tears, and repeatable identification of individual splices.

Changes in the Congressional budget in 2007 led to loss of DOE funding for year three of the three-year project. Accordingly, we have focused on a subset of these goals.

- We have completed additional software development, system testing, overall product refinement, and initial deployment through use of a reduced cost, PC-based prototype.

- We have developed computer-vision algorithms to detect vulcanized splices in material conveying belts, with the goal of alerting mine management to specific belt problems areas prior to failure. This will help eliminate catastrophic belt failures by providing managers with the information necessary to schedule maintenance activities during planned maintenance periods.

- We have developed a low-cost Smart Camera based system suitable for commercial production. The availability of a low-cost belt inspection system will allow the system to be deployed in a larger number of mines, and on a larger number of belts within each mine, providing predictive maintenance capabilities for a much larger range of mine activities, and increasing system benefits. Estimated cost for the Smart Camera based system is well below the $\$ 25,000$ target.

Patent protection for the belt inspection system has been granted by the US Patent Office under patent number 6,988,610. Over the course of the project, more than 5 million cumulative miles of belt have been inspected by deployed evaluation systems. In addition to providing unparalleled data on the appearance and evolution of splice images, these installed systems provide important experience with system reliability and usability, and have a daily positive effect on operations in the host mines.

\section{Experimental}

\subsection{Mechanical Splice Detection System}

The existing mechanical splice detection system was extended improve system effectiveness while decreasing system cost, maintenance requirements, and fragility as described below.

\subsubsection{Test Facility}

An above-ground test system was fabricated in order to allow convenient testing and refinement of the mechanical splice detection prototype. The system includes a working conveyor belt of full 60 -inch width, containing several mechanical splices. In addition, the belt contains two vulcanized splices for use in vulcanized splice detection algorithm development (see Section 3.4). The test system is shown in Figure 1. 


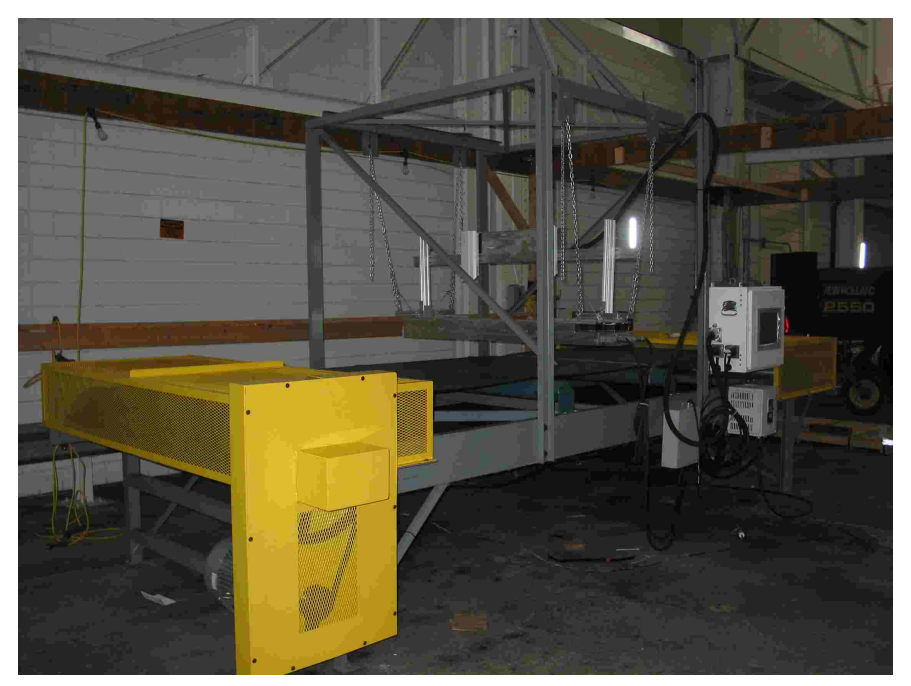

Figure 1: A full-sized test rig has been built and installed at NREC for testing and algorithm development.

\subsubsection{Design of PC-Based BeltVision Prototype}

The original belt inspection prototype system was a single, monolithic structure, involving custom fabrication and electronics assembly. This prototype is illustrated in Figure 2. This images shows the prototype in use at an underground coal mine near Blacksville, West Virginia.

In order to reduce costs and allow preliminary marketing, a low-cost PC-based prototype of the BeltVision system was developed using only off-the-shelf hardware. The frame and support structure was constructed from inexpensive, commercially available aluminum extrusion. Sensitive electronics and computing hardware were relocated to a remote enclosure, decreasing vibrational stresses and increasing reliability. Finally, the system software was updated to increase the effectiveness of operation from the surface (outside the mine). Software updates included improved capabilities for capturing and recording full-resolution belt scans to hard drive, and improved interface software to allow project personnel and end users to conveniently review the collected data, image processing results, and resulting splice classification. This software was developed with input from potential users, and was deployed at more than a dozen mines in conjunction with PC-based BeltVision systems.

The low-cost prototype includes inexpensive off-the-shelf computing hardware and a redesigned lightbar. Figure 3 shows these components, along with the protective enclosure for the computing subsystem. As with the original BeltVision system, a touchscreen user interface is provided for convenient user interaction. This PC-based prototype system has several advantages over the original design, including greatly reduced cost, increased reliability, increased portability, and increased modularity in case of hardware failure. The primary limiting factor in portability of the PC-based prototype system is the length of the light bars, which are more easily transported in a minivan than in a sedan. PC-based BeltVision systems can be rapidly assembled and deployed, and were central to the success of the project.

Figure 4 shows a schematic of a PC-based BeltVision system, while Figure 5 shows one of these systems installed on an above-ground test belt. Each BeltVision system includes a pair of 


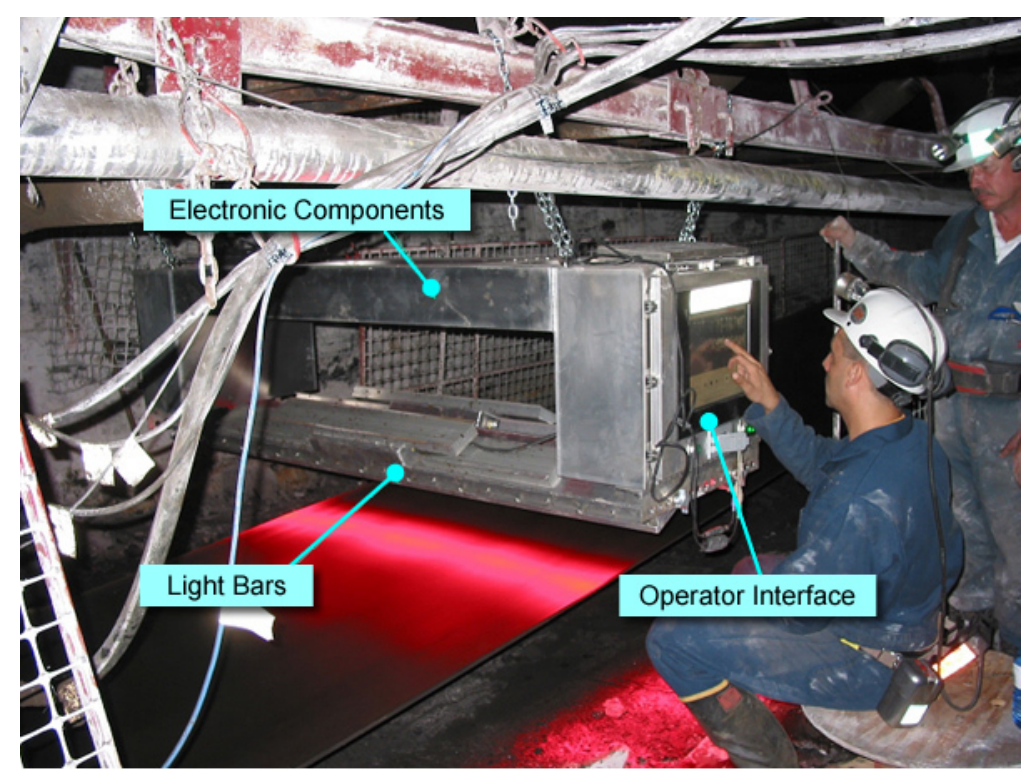

Figure 2: Original belt inspection prototype system installed in an underground coal mine.

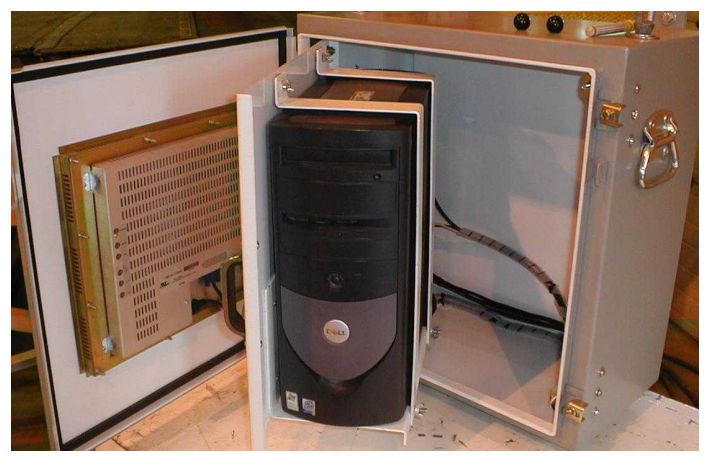

(a)

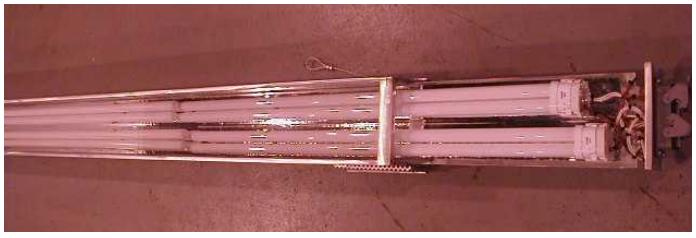

(b)

Figure 3: A reduced cost BeltVision prototype was designed in order to facilitate testing, marketing, and deployment. The new design is highly modular, permitting easy replacement of parts. Subfigure (a) shows inexpensive, off-the-shelf computing hardware in a protective enclosure, and Subfigure (b) shows a fluorescent lightbar assembly. Note that the fluorescent light bar was subsequently replaced by an LED based unit. 

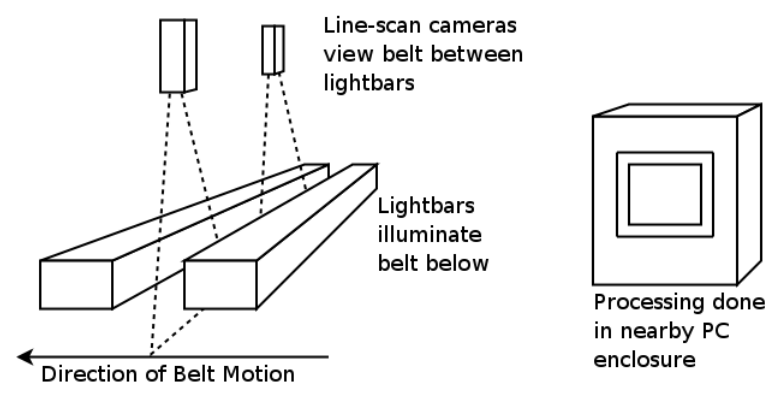

Figure 4: A schematic of the PC-based BeltVision system. For photos of the actual system, please refer to Figure 5.
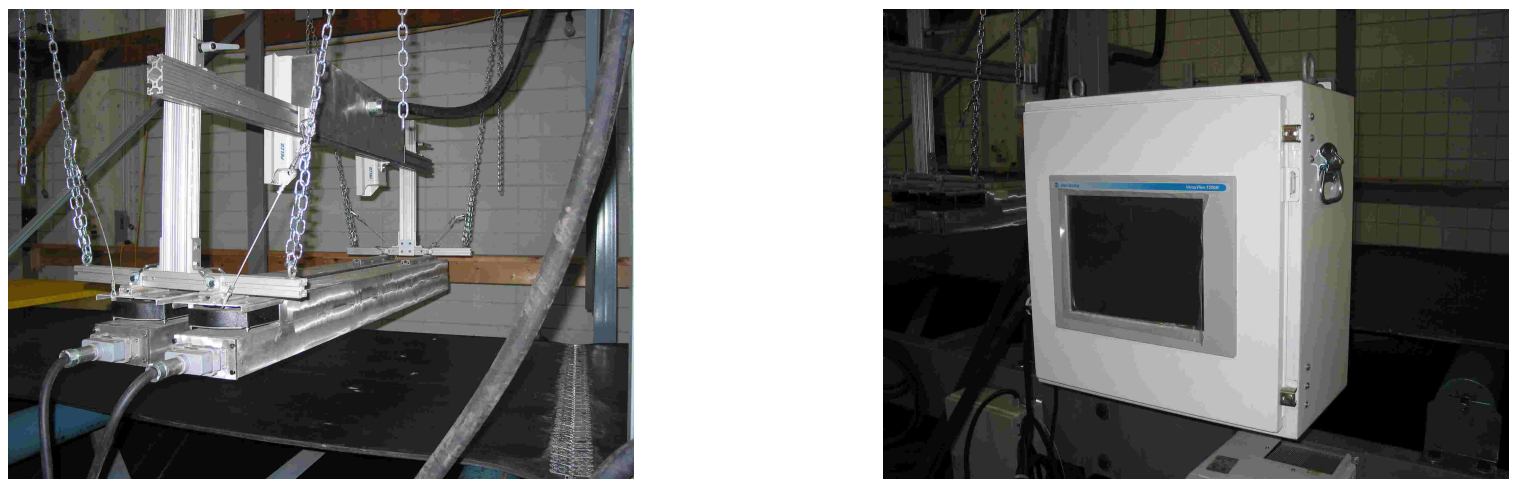

Figure 5: The PC-based BeltVision system includes two lightbars which illuminate the belt as it passes underneath the system. A pair of line-scan cameras acquire images of the belt, while processing of the acquired image data is performed in a nearby enclosure containing a commercial PC computer.

synchronized high-speed high-resolution line-scan cameras mounted side-by-side so that the output from the two cameras can be concatenated to approximate a single scan-line. The BeltVision system is positioned in such a way that the direction of belt travel is perpendicular to the camera scan-line. As the belt moves under the cameras, successive scan lines are assembled into $2 \mathrm{D}$ images as shown in Figure 6. These images are saved to hard disk and provide a high-resolution record of the belt surface. The approximate resolution of the surface image is 50 pixels/inch.

\subsubsection{Lighting Redesign}

Initial versions of the PC-based BeltVision systems used fluorescent lighting to illuminate the belt. These light bars were designed after testing a range of available lighting technologies, including multiple halogen incandescent lights, fluorescent aperture lights, and other illuminants. Ultimately, solid state lightbars assembled from off-the-shelf red LED illuminators were selected because of their observed lighting performance, moderate cost, cool operation, and mechanical robustness. 


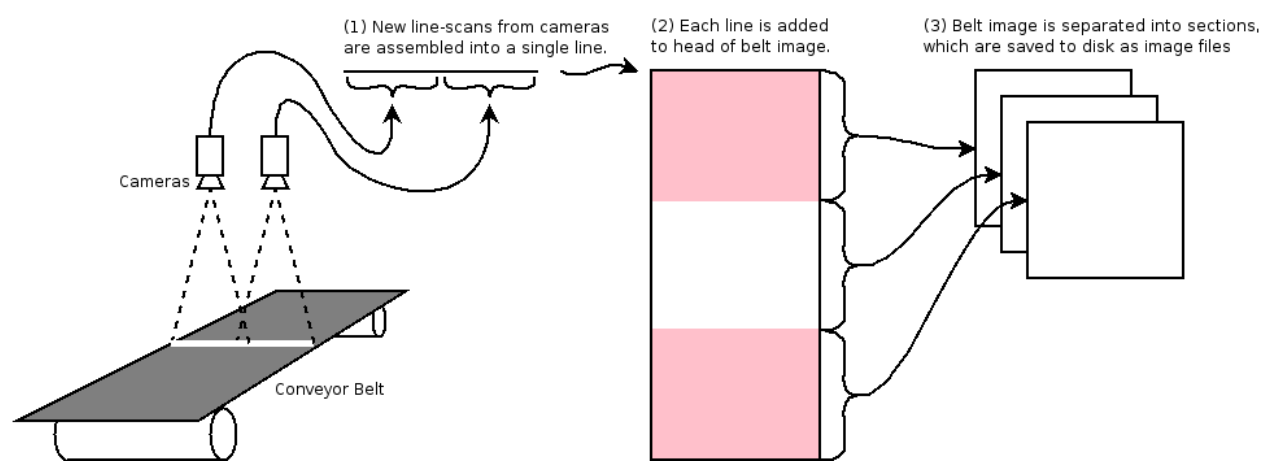

Figure 6: Individual scanlines are assembled into 2D images, which are saved to disk.

\subsection{Smart Camera Development}

The PC-based BeltVision prototypes were built using off-the-shelf cameras, frame-grabber cards, and computers. This approach allowed the system to be developed and deployed quickly, enabled rapid software development, and was critical for effective testing and system shakeout. The PCbased system has, however, it has several drawbacks for commercial use:

Expense: The frame grabber cards and cameras are still too expensive for the target market, adding thousands of dollars to the price of the system.

Reliability: Packaging a commercial PC for use in an active mine is quite difficult. The environment in the mine is harsh, dirty, and dangerous. The computers are installed in totally enclosed cabinets, which makes it a challenge to cool them properly. These factors combine to significantly increase the maintenance requirements of the system.

Size and weight: The installation sites for the BeltVision units are often far underground, and access often requires travel by foot for some portion of the journey. In the current system the enclosure for the PC is large and heavy. This adds significantly to the difficulty of both routine and unscheduled maintenance

For these reasons, a second round of redesign was undertaken in preparation for marketing and commercial use of the technology. The goal of this redesign was to continue size and cost reductions, while further increasing modularity, and hardening the system. The idea is to integrate high-resolution sensing and processing into a simple Smart Camera assembly that can be built for under $\$ 2000$.

A low cost imaging specification document was generated in order to guide selection of appropriate sensing and computational elements, and commercially available line-scan imaging elements were evaluated. Figure 7 shows sample images from several such sensing elements. A final down-select was made to a Smart Camera design based on CCD linescan element manufactured by Perkin-Elmer, an Altera FPGA and a Motorola Coldfire CPU. The overall architecture of the Smart Camera is illustrated in Figure 8. This design is based on feedback from potential users and mining experts, and strikes a good balance of functionality and overall system cost. 


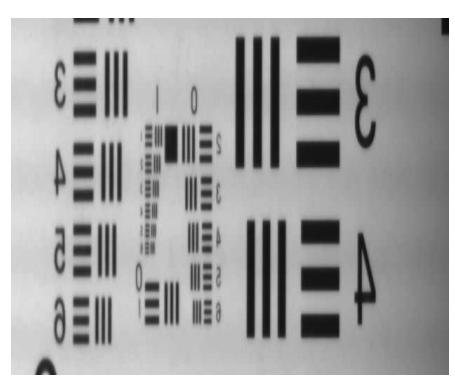

(a)

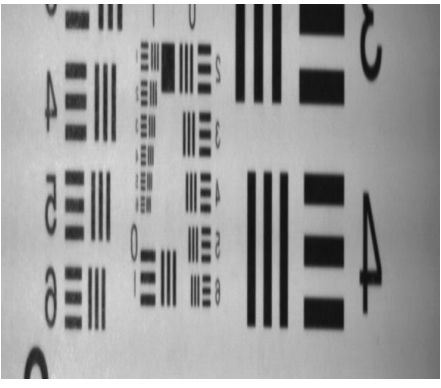

(b)

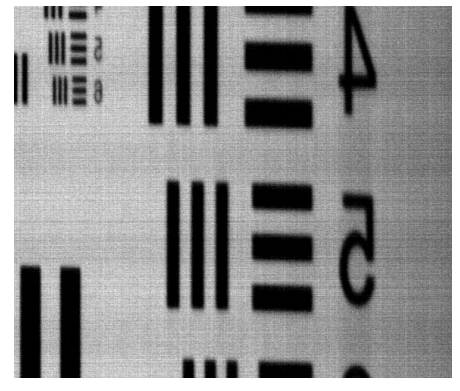

(c)

Figure 7: Several different line-scan sensing elements were evaluated: (a) shows an image from a Dalsa CCD-based line-scan element; (b) shows a similar image from a Perkin-Elmer CCD-based line-scan element which is significantly less expensive; and (c) shows an image from a CMOS line-scan element, which was even less expensive, but which was found to have inadequate image quality.
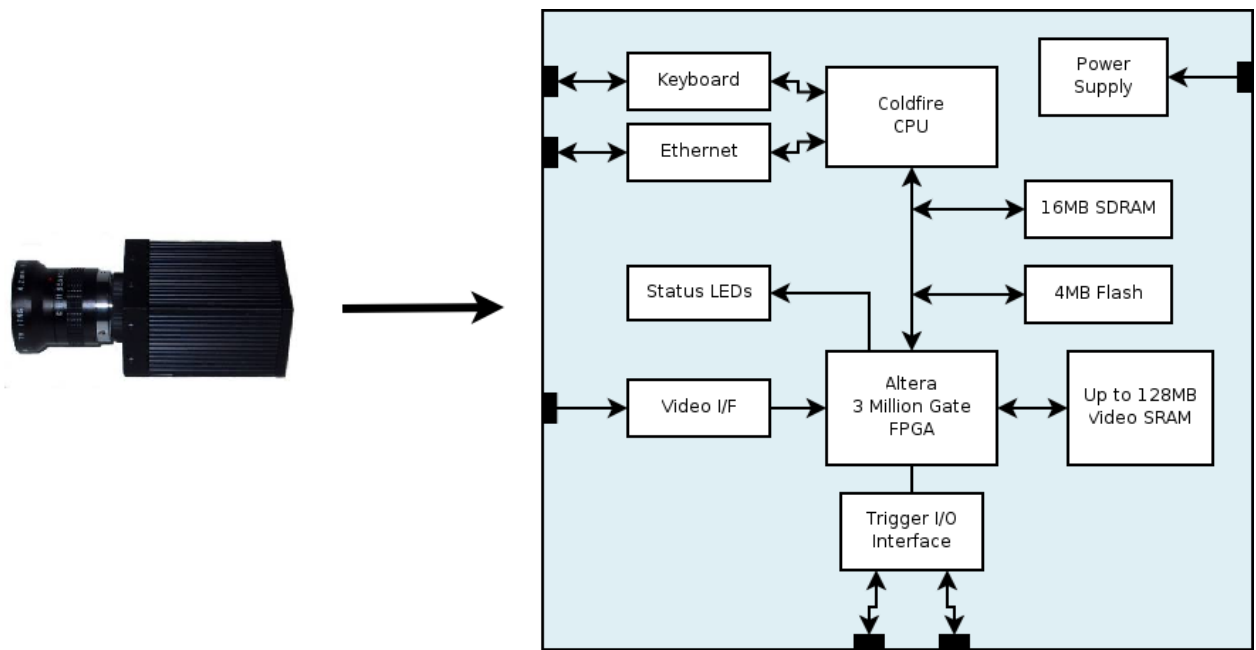

Figure 8: Overview of the initial low-cost Smart Camera system design. This illustration shows the sensor element integrated with the camera housing. In the final system, the sensor (a line-scan CCD array) is housed separately in a remotely attached sensor head, so that multiple CCDs can be driven by a single Smart Camera. 


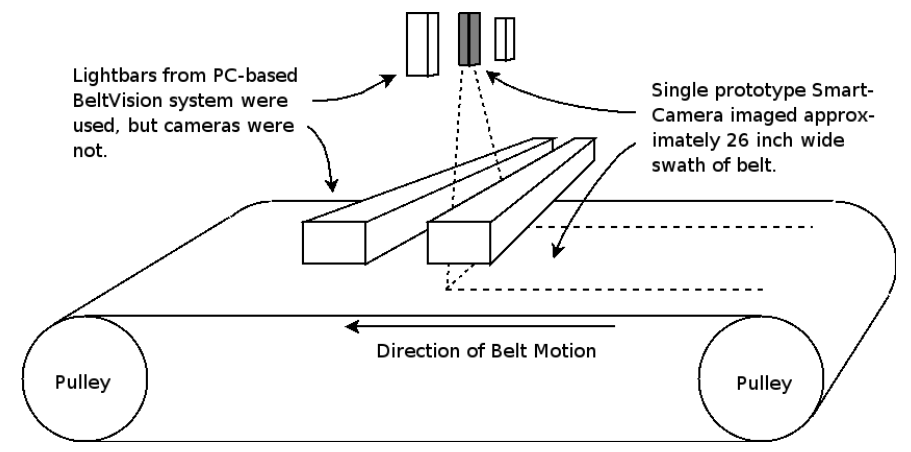

Figure 9: A schematic showing the mounting position of the Smart Camera prototype during the proof of concept demonstration.

\subsubsection{Completion of Laboratory Prototype}

A very early laboratory prototype of the Smart Camera was fabricated. The entire unit was about the size and weight of one of the the Dalsa cameras used in the PC-based BeltVision system, weighing under $5 \mathrm{lbs}$, and was easily mounted to the camera mounts of the PC-based system for testing.

Both a PC-based BeltVision System and the prototype Smart Camera were attached an the above-ground test rig. The Smart Camera was mounted mid-way between the positions of the two cameras in the PC-based system, and oriented so that its scan line imaged approximately 26 inches of belt between the two lightbars. In order to visualize this setup more clearly, please refer to Figure 9. The test rig was very similar to the one shown in Figure 1, and had a short (approximately 20 meter) length of belt containing several mechanical splices. When the motor drive was activated, the belt moved under the mounted BeltVision unit, simulating the motion of the much longer belts at the mine installation sites.

The light bars mounted on the test rig were activated and the belt was run at speeds varying from $1 \mathrm{~m} / \mathrm{s}$ to $5 \mathrm{~m} / \mathrm{s}$ over the course of approximately 45 minutes. During this time, the prototype was carefully observed to ensure that there were no failures in mechanical splice detection.

\subsubsection{Final Design and System Implementation.}

Based on testing of the initial Smart Camera prototype and assessment of software performance, the on-board FPGA was upgraded to a more powerful Xilinx chip, and on-board memory size was increased. These changes allowed several important additions to the functionality of the system:

- Improved handling and buffering of image data, including JPEG compression and decompression on-the-fly.

- Ability to simultaneously interface with two line-scan sensing heads. This allows the entire width of the conveyor belt to be imaged by a single Smart Camera, and further decreases total system cost. The two sensing heads are connected to the Smart Camera body cables, and can be mounted independently.

- Additional image alignment routines so that data from the two sensing heads can be easily integrated. 


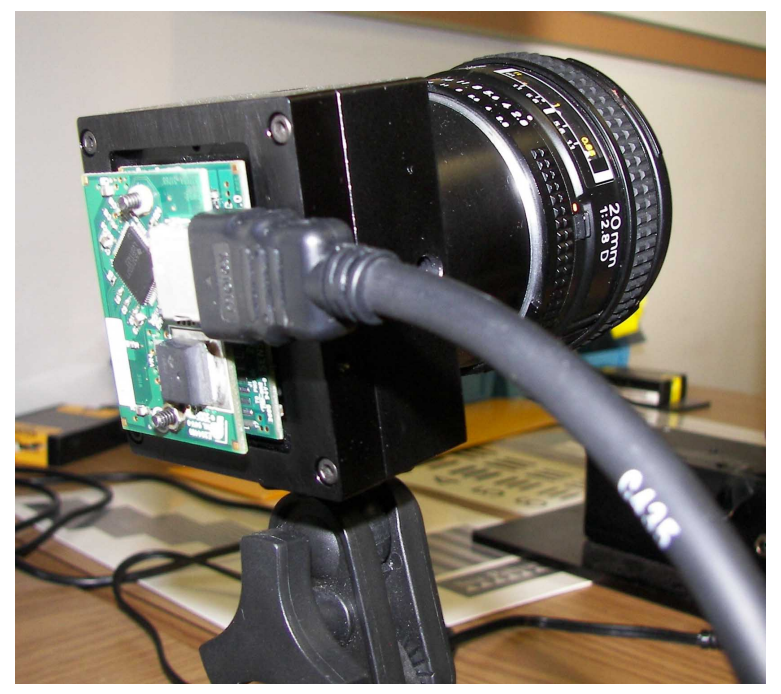

Figure 10: A partially assembled camera head for the Smart Camera based BeltVision system.

- Improved communication with supervisory PCs via Ethernet to allow numbering of splice images, control of detection parameters, etc.

Housings for the camera-head assemblies were designed, and camera-head enclosures were fabricated. Figure 10 shows a camera head with its back cover removed, and with sensing element and I/O board installed. These camera heads will replace the expensive commercial line-scan cameras in the current BeltVision prototype, and connect to the FPGA housing using an inexpensive HDMI cable.

A photo of a fully assembled and functional FPGA board is shown in Figure 11.

\subsubsection{Embedded Software Development}

The image data pathway from the sensors, through the FPGAs to video memory was finalized and implemented. The FPGA code originally developed for the 400,000 gate Altera FPGA was ported to run on the Xilinx FPGA, and additional code was written to support more flexible image output, reporting of splice detection results, and buffering of image data.

Still more FPGA software was developed to perform JPEG compression and decompression of the image data. Compression is required in order to reduce video memory requirements. Decompression is required for the local display, and must occur concurrently with the JPEG compression. Code was also developed to allow horizontal and vertical offset image adjustments so as to match up the scan lines from two individual camera heads as if they came from one camera head with an extra-wide field of view.

Software was developed to allow interfacing with the Smart Camera over Ethernet, and code was written to implement the image annotation and display features called for in the image path design. This code includes software to manage a local LCD display and touchscreen.

The system was bench tested in September, 2006. Camera input was simulated by moving pictures of mechanical splices past a single sensor head, and splice detector functionality was monitored via PC, as shown in Figure 12. Internal software diagnostics indicated correct functioning of 


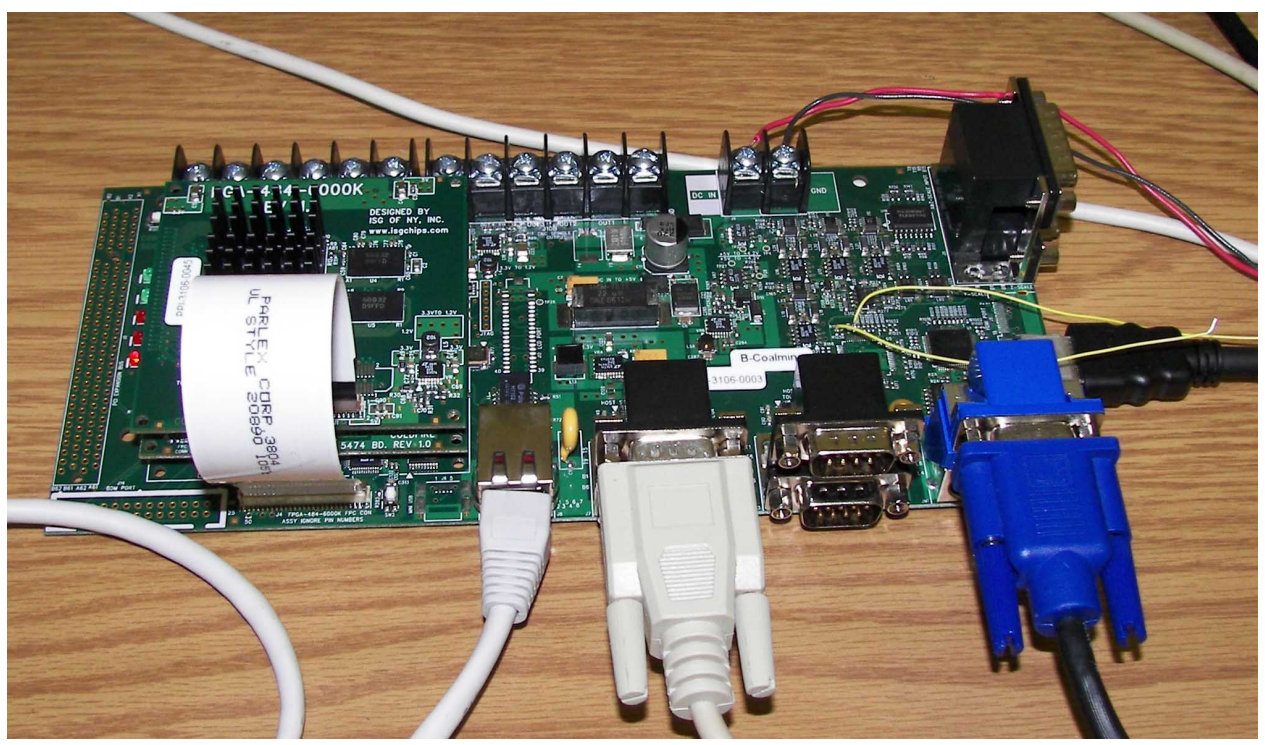

Figure 11: A fully functional computing board for the Smart Camera, photographed during demonstration in September, 2006.

the splice detection algorithm, and qualitative performance was deemed acceptable.

Finally, FPGA output was integrated with the control and communications software so that full-resolution images of detected splices can be sent to the surface via Ethernet. The result of this effort is a system in which all of the the sensing, processing, and communications are carried out by small, lightweight, inexpensive hardware modules. System maintenance consists of simply unplugging any malfunctioning units and replacing them with parts from a backpack or box.

The system was packaged for underground installation, and a fully packaged unit was deployed at the Bailey Mine near Washington, PA. The Smart Camera enclosure is more than 10 pounds lighter than the PC-based unit from the previous prototype, and can operate with no moving parts or disk drives to reduce reliability. Another major advantage is that Smart Camera hardware has significantly lower power consumption. This allows the sealed Smart Camera enclosure to run continuously without significant heat buildup.

\subsection{Infrared Detection of Vulcanized Splices}

Although the primary focus of early efforts involved detection of mechanical splices, vulcanized splices are used on a wider range of belts in mining and material handling applications. Vulcanized splices are formed by fusing disjoint sections of belt together under heat and pressure. The layers of fabric that make up the conveyor belt core are not continuous through the splice. Vulcanized splice failure typically begins with delamination of the joined sections of the belt. The delamination worsens with use, eventually leading to splice failure. This progression suggests that the extreme variations in belt tension as the belt moves from a loaded section of its run to an unloaded section (or vice versa) may be repeatedly stretching the vulcanized rubber and fabric, eventually leading to fatigue and failure.

Experiments were conducted to evaluate the extent to which this stretching, and concomitant 


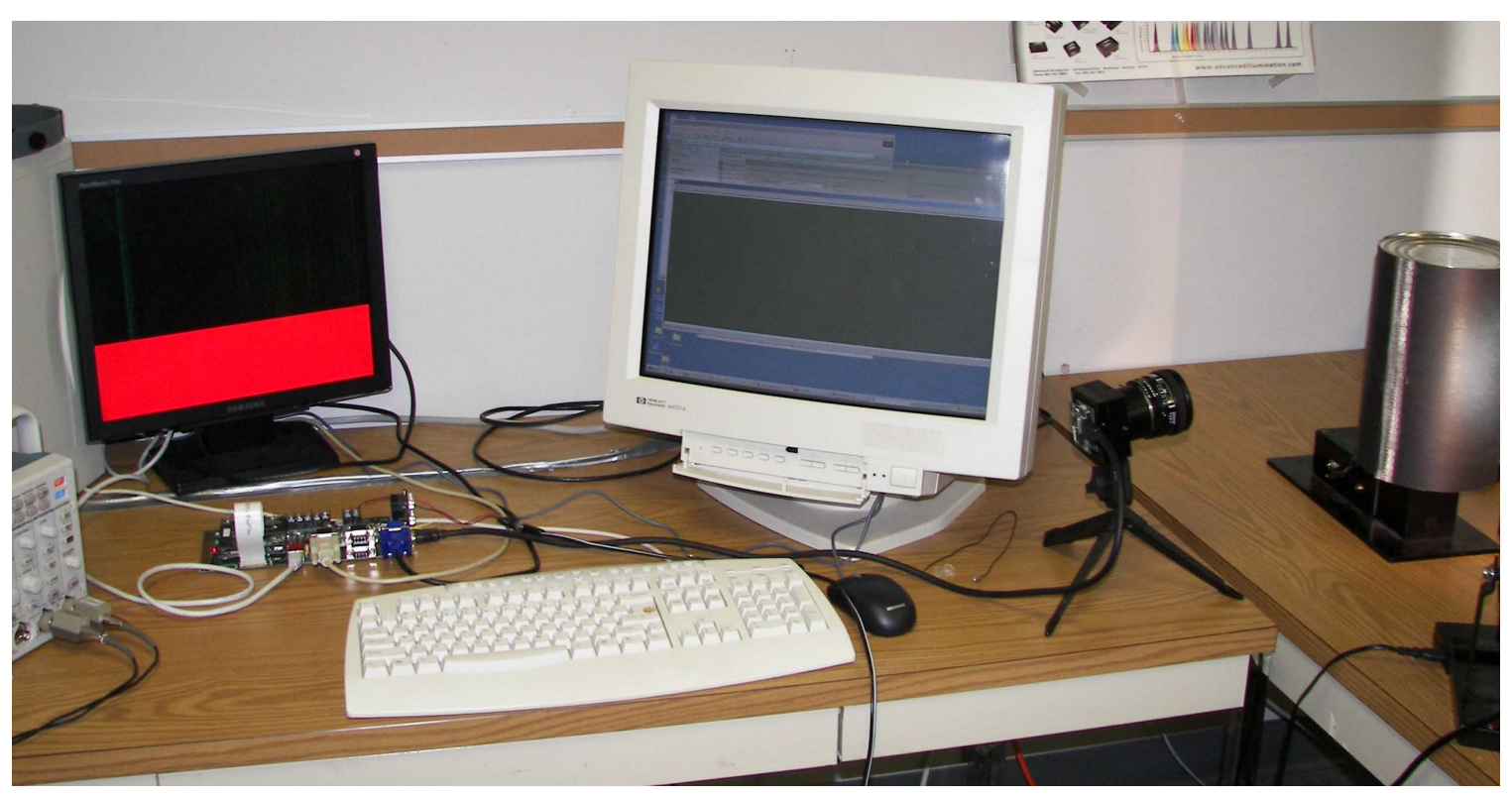

Figure 12: A bench test of a single-headed Smart Camera system was conducted in late 2006.

heating, affect the surface temperature of the belt. This investigation was conducted on the grounds that significant heating would provide an easily detectable marker for vulcanized splices, particularly those splices that are beginning to fail.

\subsubsection{Experimental Apparatus}

An infrared thermographic camera (Thermovision A series, manufactured by FLIR Systems) was temporarily obtained. The camera was mounted in two active mines: the Loveridge mine near Fairview, West Virginia; and the Blacksville mine near Blacksville, West Virginia. In each case, the camera was positioned so as to image the belt as it passed over a main driver roller, since this is a point of transition between high and low tension sections of the belt. In the Loveridge mine, particular attention was made to be sure that a length of belt was visible downstream of the transition area. In both mines, the camera output was connected to a laptop computer so that temperature measurements could be recorded to hard drive.

Discussion of the infrared detection system is continued in Section 4.3.

\subsection{Vision-Based Detection of Vulcanized Splices}

When sections of conveyor belt are joined using vulcanized splices, the ends are cut at an angle. For the mines involved in this study, the cut angle is nominally $22^{\circ}$ and the junction between the belt sections produces a characteristic diagonal line across the surface of the belt. A vulcanized splice detection algorithm has been developed that operates on 2D visual images of the conveyor belt by searching for these lines. The 2D images are acquired from line-scan cameras mounted above an unloaded section of belt, just as described for mechanical splice detection in Section 3.1. Accurate line detection and classification is challenging because of several factors: the apparent line angle in the belt images depends on the speed of the belt and the line-scan frequency; splice lines are often 


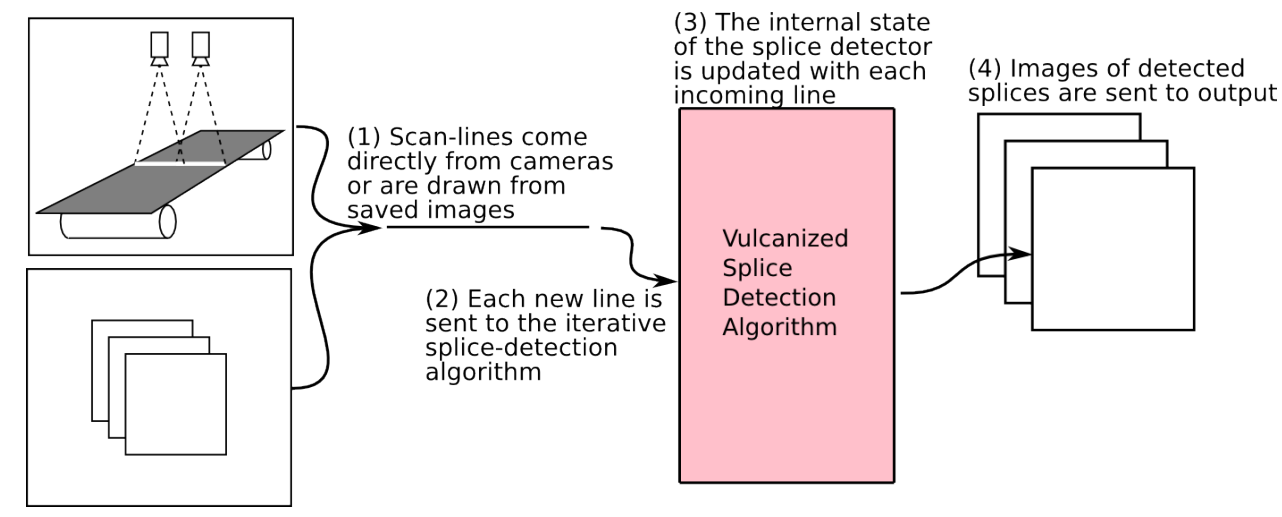

Figure 13: The vulcanized splice detector allows incremental processing of image data. In the current installations, direct connection between the cameras and the vulcanized splice detection software is not implemented. Instead, splice detection is automatically performed twice a day using images which have been saved to hard disk. as shown in Figure 6.

very faint; and typical belt images include spurious lines caused by water, pulley lagging, internal belt structure, and other artifacts. The following subsections describe this research and development effort.

\subsubsection{Experimental}

The splice detection algorithm is designed to allow incremental processing of image data as illustrated in Figure 13. Scan-lines are sent to the detection algorithm one-by-one, and each vulcanized splice is flagged as soon as it is detected. Ultimately, camera output will be sent directly to the splice detection algorithm. During the course of this project, complete scans of the belt surface were saved to a computer hard drive twice a day, and these saved images were used as input to the vulcanized splice detector. Images of detected splices were sent to the surface via Ethernet, where they were reviewed by the belt foreman to monitor splice condition. This process is automated and runs without any user intervention beyond adjustment of detector sensitivity. It is not practical to transmit the complete scan of the belt to the surface, due to the bandwidth limitations of the local network.

Figure 14 shows a graphical user interface used to monitor algorithm performance during early development. Note that in actual use, the algorithm runs unattended, with no GUI, and simply saves its results to disk.

Discussion of the vision-based vulcanized splice detector is continued in Section 4.4.

\subsection{Lighting Refinements}

During most of the project period of performance, the belt inspection system system included two LED light bars, suspended above the belt, one upstream of the cameras, and one downstream of the cameras, as shown in Figure 15(a). Subsequent testing has shown that splice recognition accuracy for both mechanical and vulcanized splice detection algorithms can be maintained using only one light bar positioned an oblique angle as shown in Figure 15(b). From this angle, the light highlights surface detail on the belt, in some cases improving system performance. This change is significant 


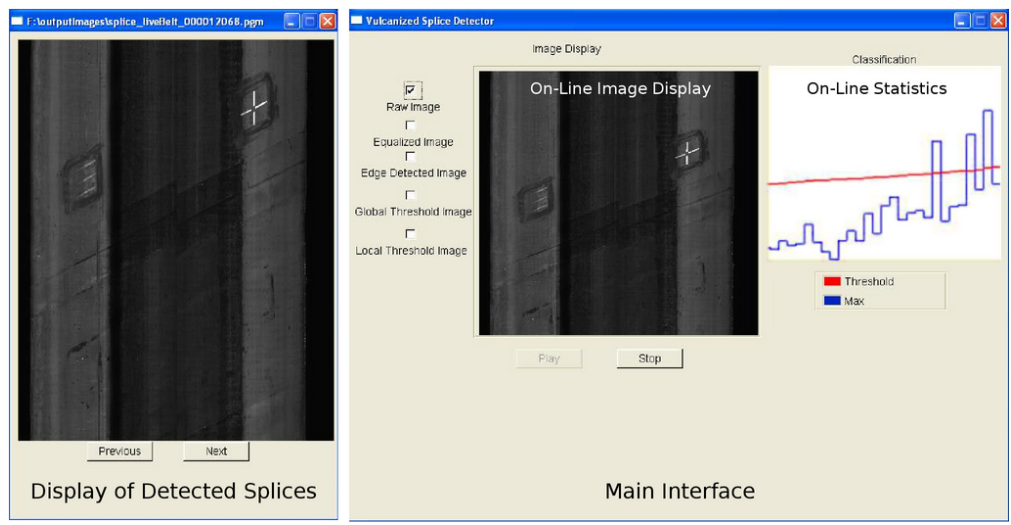

Figure 14: A screenshot of the GUI-based vulcanized splice detection application under development. Note that the inclusion of white fiducial markings in the neighborhood of vulcanized splices is very rare: the white cross visible in the screenshot is highly unusual.

not only as a cost reduction measure, but also because it halves the expected number of lighting component failures, and reduces system setup time. Extended deployment tests are underway to determine whether the new position affects the incidence of fouling or mechanical damage to the lightbar.

\section{Results and Discussion}

\subsection{Mechanical Splice Detection System}

Fourteen PC-based BeltVision systems have been installed in active mines throughout western Pennsylvania and West Virginia, and an additional two units are installed in customer mines outside this region. These sixteen systems are in active daily use and are currently having direct positive impact on mine productivity and worker safety.

\subsection{Smart Camera Development}

In preparation for deployment of the new Smart Camera based BeltVision system, the entire system was subjected to rigorous bench testing, and demonstrated on the above-ground conveyor test setup. Images from this demonstration are shown in Figure 16. After initial configuration, the mechanical splice detection algorithm was run under human observation for three separate tests, each time detecting at least 48 mechanical splice transitions with no missed detections and no false positives.

The Smart Camera system is currently undergoing shake-out testing in preparation for initial low volume marketing. Figure 17 shows the fully packaged prototype as deployed in the Bailey mine, near Bailey, West Virginia.

Underground installation of the Smart Camera is still fairly recent, so reliability statistics are not yet complete. Initial results are promising, with the Smart Camera hardware providing a drop-in replacement for the larger, heavier, more expensive PC-based prototype. Because the Smart Camera does not require a hard disk drive for operation, and because of the low-power, low-temperature, unvented operation of the Smart Camera, we expect significantly improved reliability. 


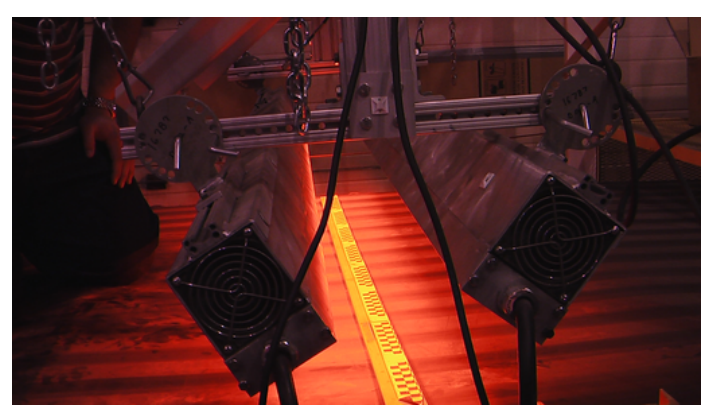

(a)

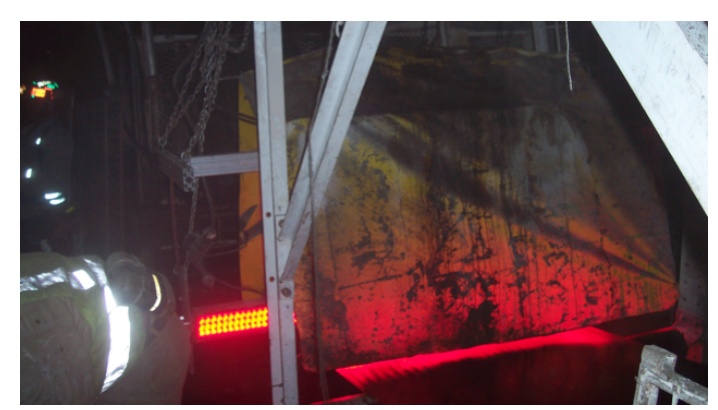

(b)

Figure 15: (a) The original lighting configuration, using two light bars, deployed at Beitzel Corporation's above-ground test facility. The photograph is taken from beside the belt, looking perpendicular to the direction of belt travel, along the length of the light bars. A yellow calibration target is lying across the width of the belt. (b) The revised, single-light-bar configuration deployed underground. The light bar is partially obscured by a piece of brattice cloth, with the LED light emitting surface facing the camera. The brattice cloth curtain significantly reduces fouling of the cameras and light bar.

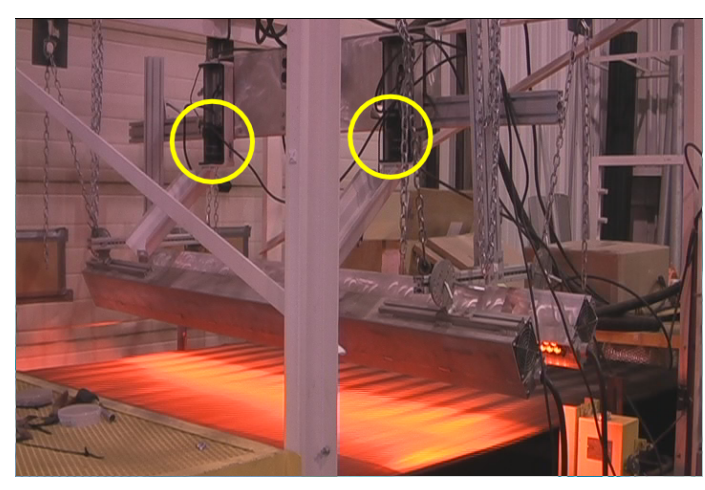

(a)

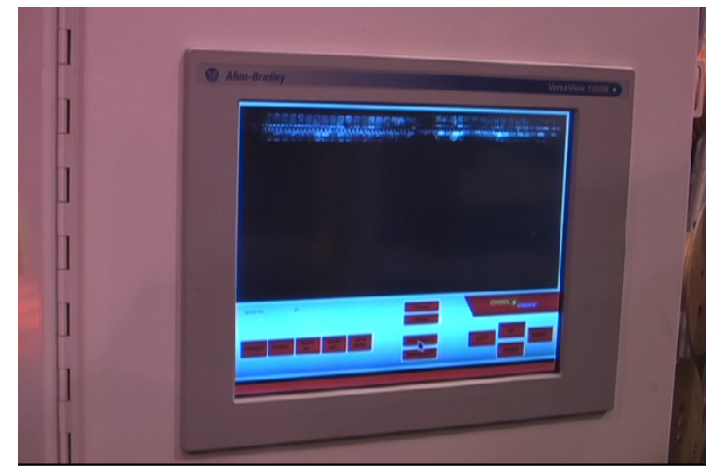

(b)

Figure 16: (a) The two-headed Smart Camera under test at the above-ground test facility. A conveyor belt passes underneath two LED light bars. The two yellow circles indicate the new camera heads, which are considerably smaller and less expensive than the Dalsa units that were previously used. (b) An LCD screen being driven by the Smart Camera. The top of the screen shows an image of the most recently detected mechanical splice, while the bottom of the screen shows an GUI overlay that can be manipulated via touchscreen. 

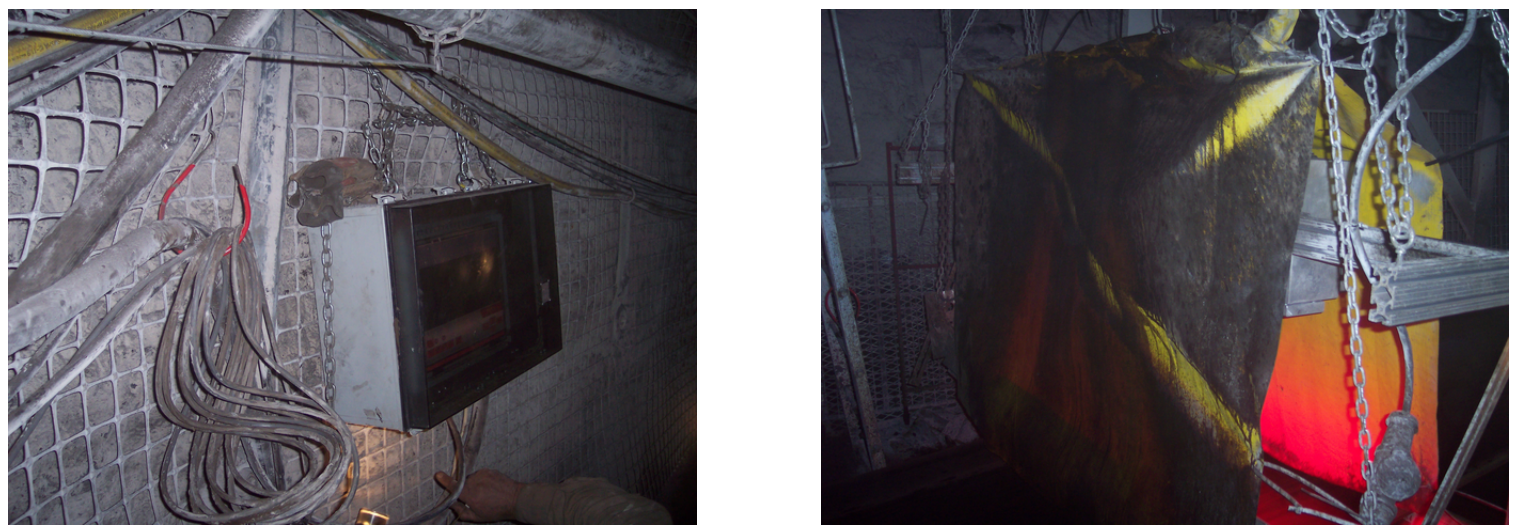

(a)

Figure 17: (a) The Smart Camera main board installed in wall-mounted enclosure in the Bailey mine for testing. The transparent cover of the enclosure folds down to allow access to an LCD touchscreen. The cover can be closed to protect the touchscreen without obscuring the display. (b) The light bar and two camera heads are suspended above the belt on an aluminum frame. This picture was taken on an operating belt, and the assembly is partially obscured by brattice cloth, which greatly reduces fouling of the cameras and light bar cover.

\subsection{Infrared Detection of Vulcanized Splices}

Using the apparatus described in Section 3.3, full resolution thermographic images were acquired for several revolutions of belts in both the Loveridge and Blacksville mines. The field-of-view and placement of the camera were such that the portions of belt visible in subsequent frames overlapped by roughly $20 \%$. Figure 18 illustrates the thermographic imaging interface during acquisition of the Blacksville belt scan. Note that the colormap of the image has been adjusted to highlight small temperature variations.

The range of temperature variation was computed for each belt, and the acquired images were analyzed to identify regions noticeably outside the norm. The working hypothesis that vulcanized splices show significant heating as they transition between loaded and unloaded portions of the belt was not borne out by the data. In both mines, no discernible temperature difference between vulcanized splices and other regions of the belt was detected, and this approach was abandoned.

\subsection{Vision-Based Detection of Vulcanized Splices}

Using PC-based BeltVision systems, full resolution recordings were acquired at seven installation sites in active mines, including the Bailey mine and Enlow Fork mine, both located on the Pittsburgh 8 Seam in Southwestern Pennsylvania; and the Robinson Run mine and Blacksville 2 mine, both in Northern West Virginia, and also located on the Pittsburgh 8 Seam. Each recording included images covering the entire belt. Footage from each site was grouped into a series of 2D images, each containing 4096 scan lines and representing approximately 82 inches of belt surface.

The images from each mine were observed to have different character, depending on both the specific belt and the environmental conditions of the installation site. Reduced resolution images from four installation sites are shown in Figure 19. All four of the images in this figure have vertical 


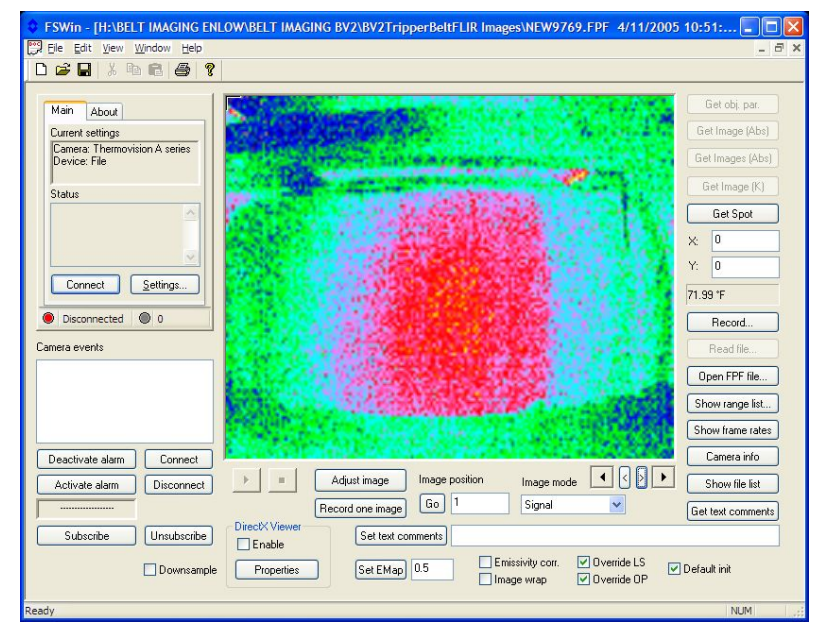

Figure 18: The thermographic imaging interface during acquisition of footage from the Blacksville mine.

banding, which is characteristic of all of the belts we have observed. Of particular interest are the diagonal lines introduced by pulley lagging in the images from the Bailey mine, since these lines are easily mistaken for vulcanized splices. Also of interest is large amount of noise in the Robinson Run images due to mud and water on the belt. In order to show these phenomena more clearly, sections from both the Bailey image and the Robinson Run image are shown at higher magnification in Figure 20. The contrast of each of the images in this Figure has been increased somewhat to provide a clearer view of the belt detail.

The belt images were manually inspected to identify vulcanized splices, and the name of the image containing each splice was recorded in a spreadsheet for use as ground truth in analyzing vulcanized splice detector performance. For those splices which overlapped the border between two images, the names of both images were recorded. Each image sequence contained between 20 and 60 splices. Figure 21 shows representative splice images from each sequence, and Figure 22 shows the same images, this time with enhanced contrast so that the detail is more readily visible. The discontinuity along the vertical centerline of the images is due to misalignment of the two cameras in the BeltVision system, and is discussed further below. This discontinuity is most clearly visible in Figure 22(b).

The vulcanized splice detection algorithm has one tunable parameter, which adjusts the sensitivity of the detector. Each dataset was processed using several values of this parameter, and the output of the vulcanized splice detector was compared with the human-scored ground truth. A splice was considered to have been correctly detected if the algorithm indicated that a splice was present during processing of the 2D image which contains that splice. Ground truth splices which overlapped the border of two images in the dataset were considered to be correctly detected if the algorithm indicated that a splice was present during processing of either or both of those images. Counts of missed splices and false positives were generated for each combination of mine and tuning parameter value, as shown in tables 2 through 5. Figure 23 shows ROC graphs for the Bailey Mine dataset and the Robinson Run dataset. No ROC graphs are presented for the Blacksville and Enlow Fork results because on both of these datasets the algorithm achieved perfect detection for some value of the tunable parameter.

The purpose of this effort was to demonstrate feasibility of vulcanized splice detection using image processing techniques. The preliminary results for both belts were wholly satisfactory. In both cases, it was possible to detect all of the known vulcanized splices without an excessive number of false-positives. Overall, more than 30 miles of belt footage were manually scored, and several hundreds of splices were labeled.

\subsubsection{Epilogue}




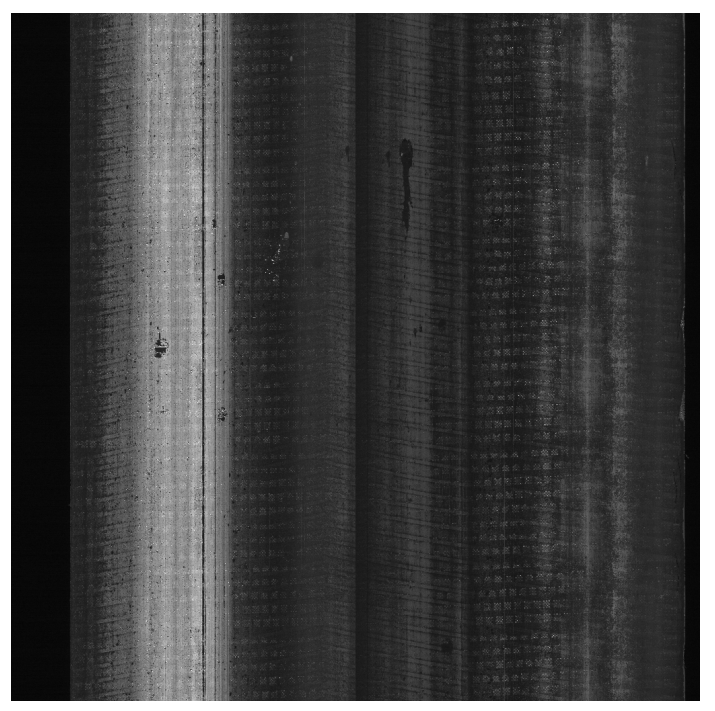

(a) Bailey Mine

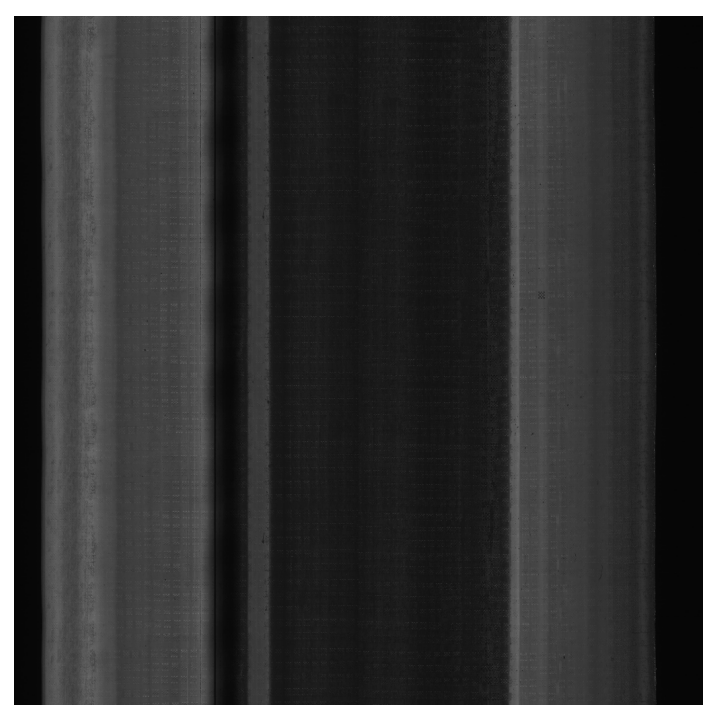

(c) Enlow Fork Mine

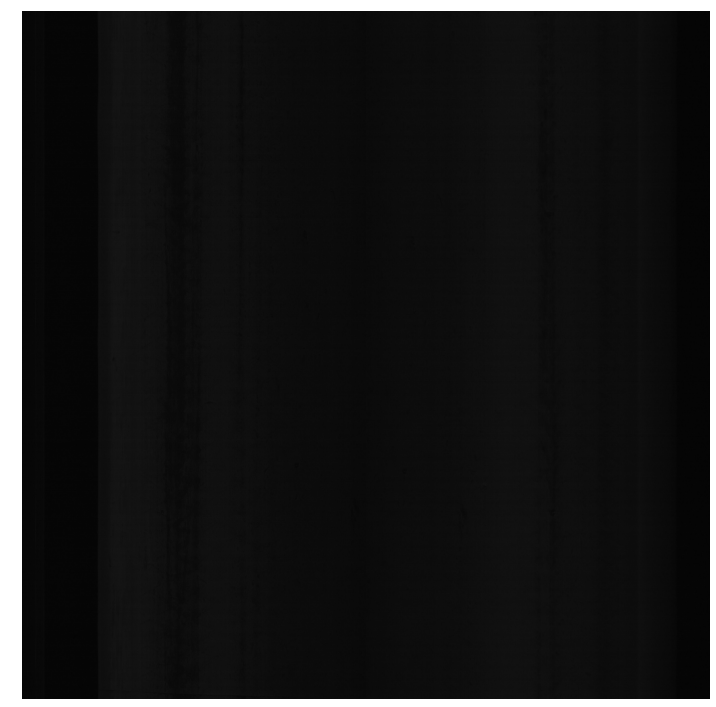

(b) Blacksville Mine

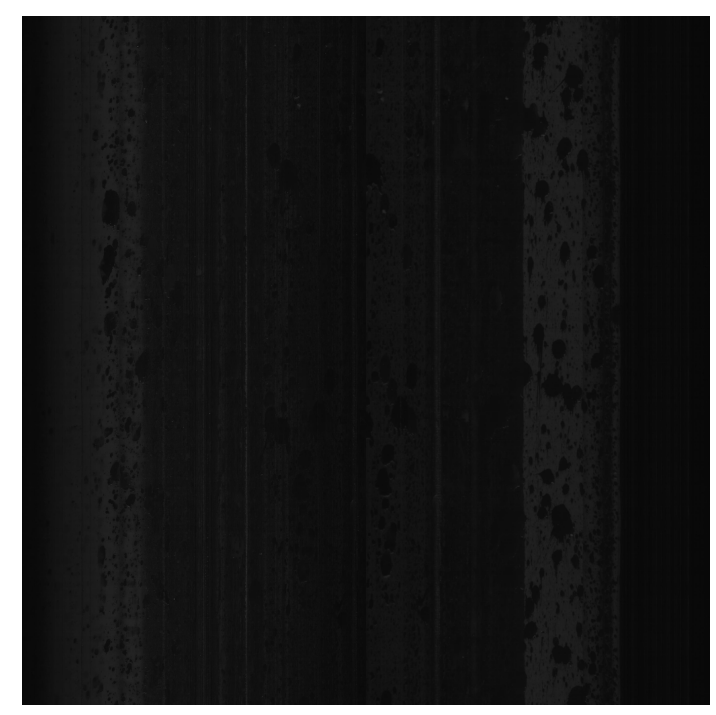

(d) Robinson Run Mine

Figure 19: Raw images from each of the four tested installation sites. 


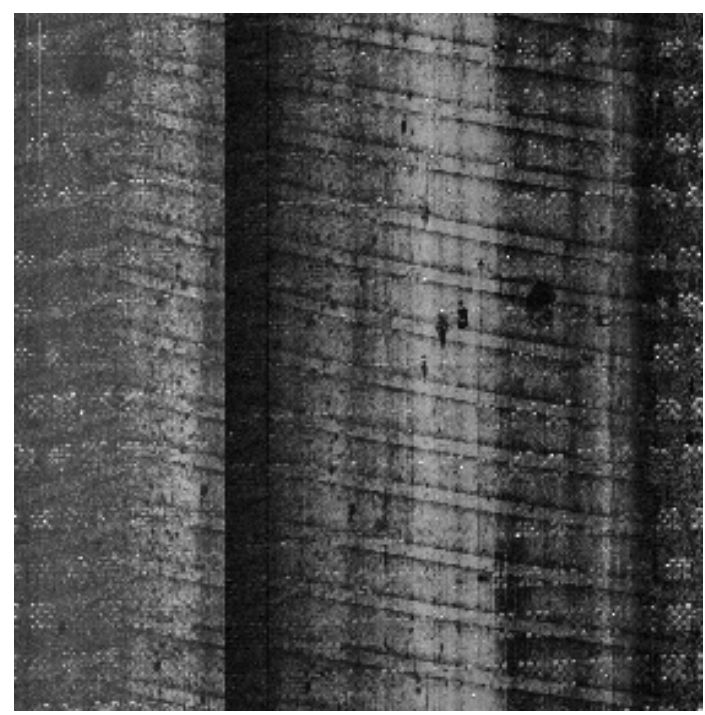

(a) Bailey Mine

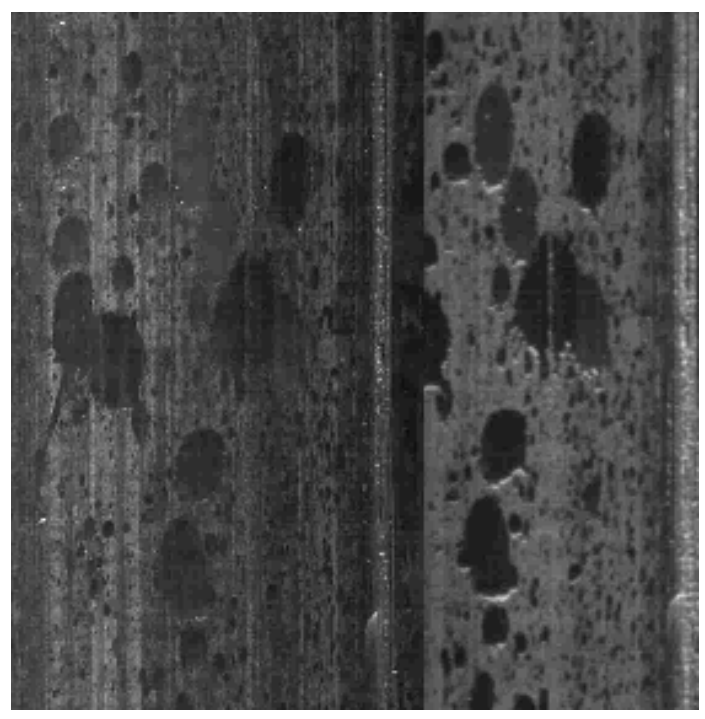

(b) Robinson Run Mine

Figure 20: Sections of the images from the Bailey Mine and Robinson Run Mine. The contrast of these images has been increased somewhat to permit easy viewing. Pulley lagging in the Bailey mine image leaves an impression on the belt which is easily mistaken for the diagonal line of a vulcanized splice.

\begin{tabular}{|c|c|c|c|}
\hline Threshold & True Positives & False Positives & Missed Detects \\
\hline 1.9 & 7 & 212 & 0 \\
\hline 2.1 & 7 & 39 & 0 \\
\hline 2.3 & 7 & 8 & 0 \\
\hline 2.5 & 7 & 3 & 0 \\
\hline 2.7 & 6 & 0 & 1 \\
\hline 2.9 & 6 & 0 & 2 \\
\hline 3.1 & 5 & 0 & 2 \\
\hline 3.3 & 5 & 0 & 2 \\
\hline 3.5 & 5 & 0 & \\
\hline
\end{tabular}

Table 2: Splice detection results the refined vulcanized splice detection algorithm applied to the Bailey Mine dataset. The column labeled "True Positives" indicates how many splices were correctly identified by the splice detection algorithm. The column labeled "Missed Detects" indicates how many images were manually determined to contain vulcanized splices, but were not identified by the detection algorithm. The column labeled False Positives indicates how many images were manually determined not to contain vulcanized splices, but were identified by the detection algorithm. 


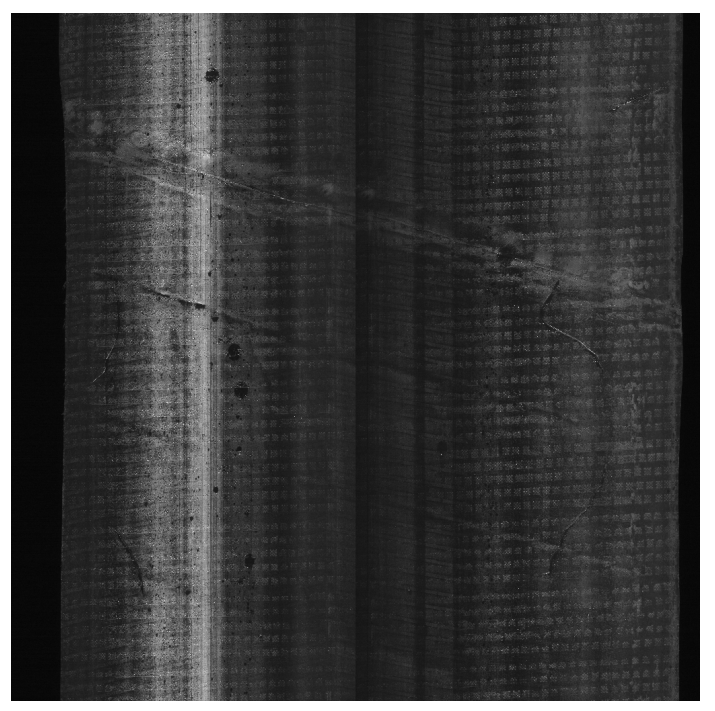

(a) Bailey Mine

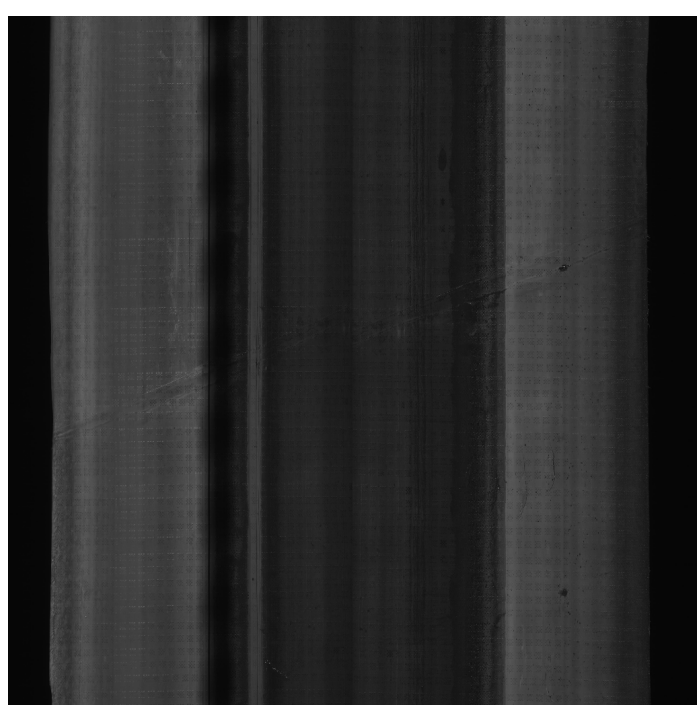

(c) Enlow Fork Mine

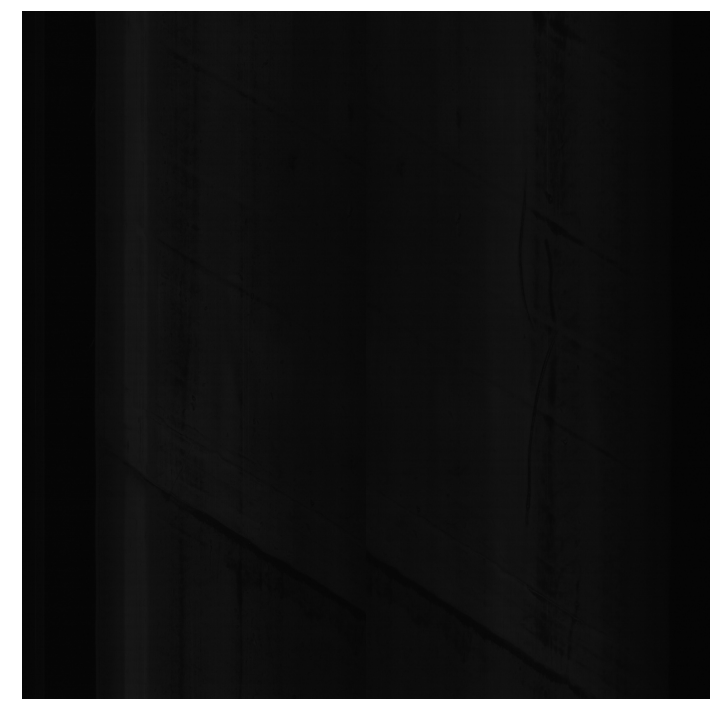

(b) Blacksville Mine

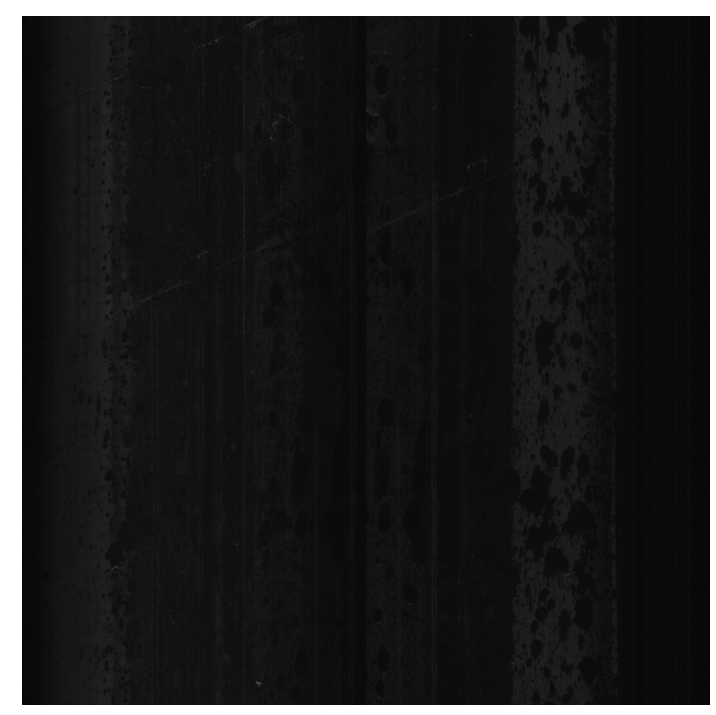

(d) Robinson Run Mine

Figure 21: Representative splice images from each of the four tested installation sites. 


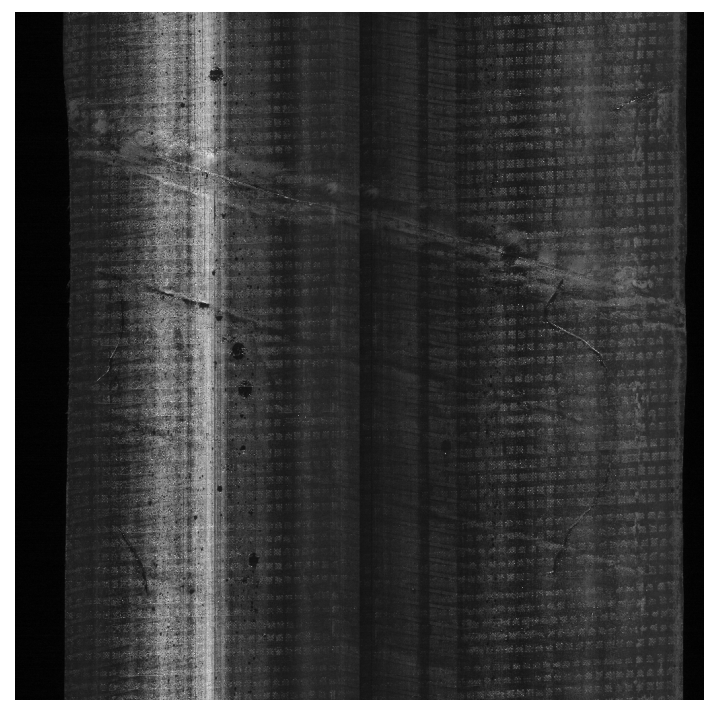

(a) Bailey Mine

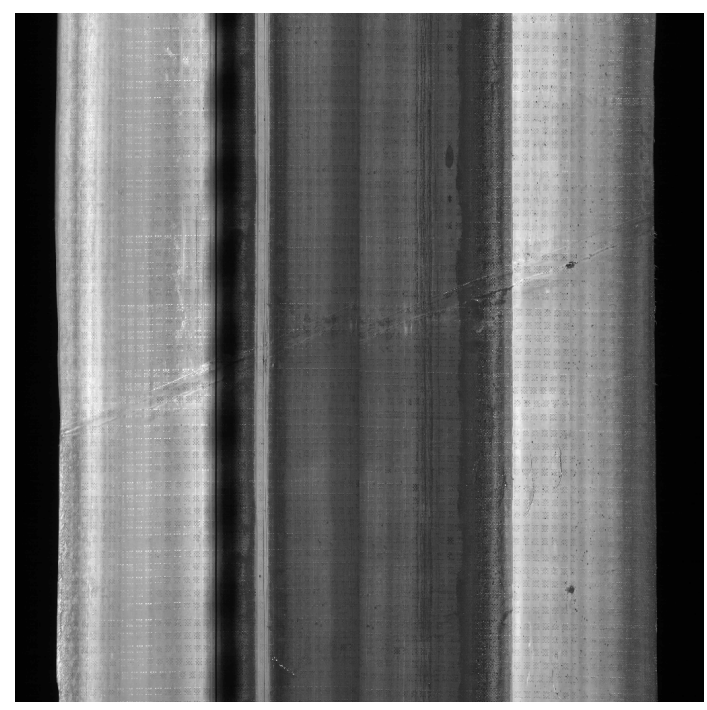

(c) Enlow Fork Mine

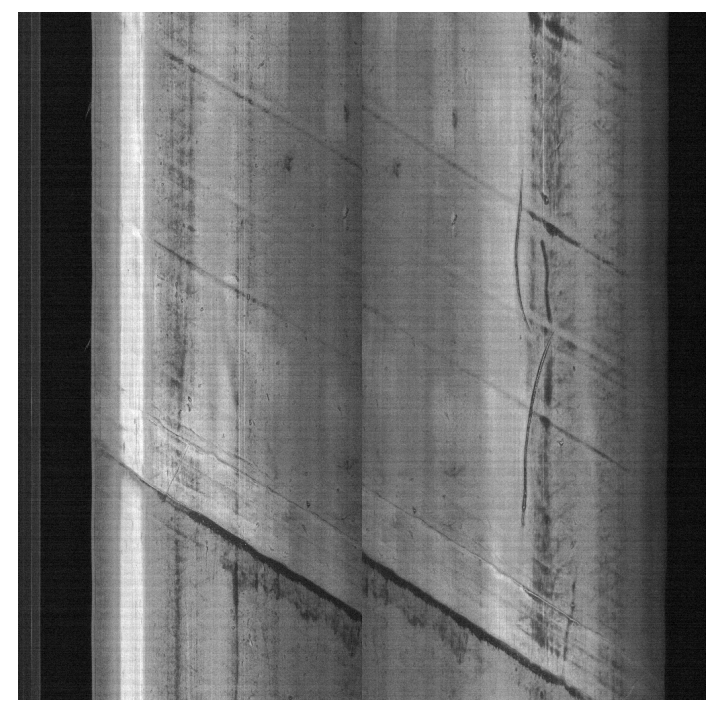

(b) Blacksville Mine

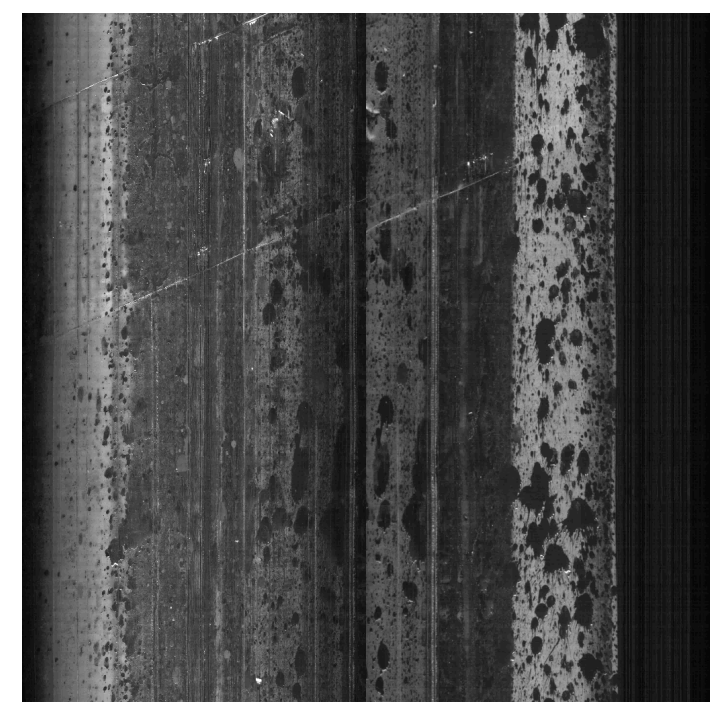

(d) Robinson Run Mine

Figure 22: These are the same images a shown in Figure 21, except that the contrast of each image has been adjusted to make the splice more easily visible. The Bailey Mine image is not visibly changed because the original image has very high contrast. 


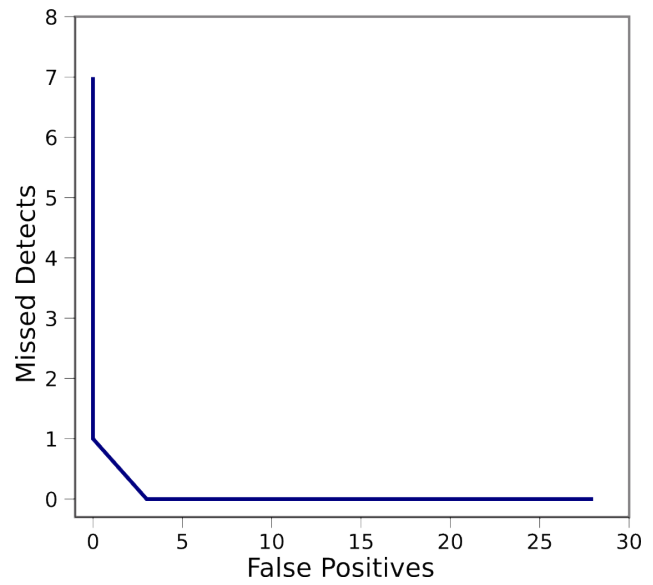

(a) Bailey Mine

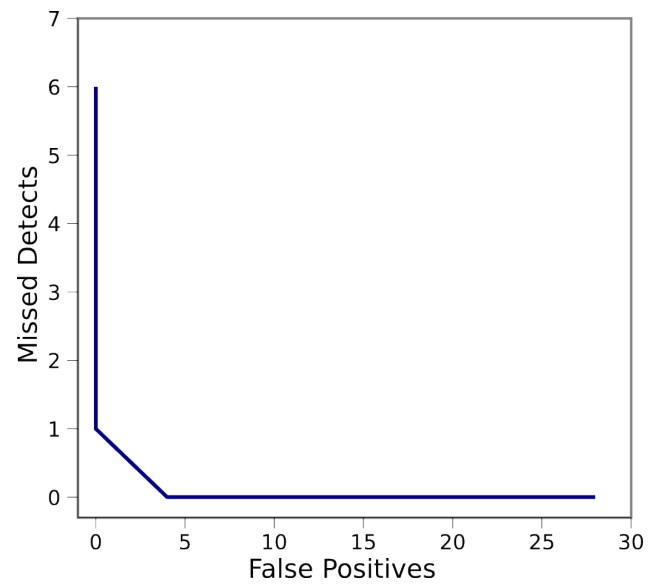

(b) Robinson Run Mine

Figure 23: ROC curves for the Bailey and Robinson Run Mine datasets.

\begin{tabular}{|c|c|c|c|}
\hline Threshold & True Positives & False Positives & Missed Detects \\
\hline 2.5 & 4 & 36 & 0 \\
\hline 2.9 & 4 & 8 & 0 \\
\hline 3.3 & 4 & 4 & 0 \\
\hline 3.7 & 4 & 3 & 0 \\
\hline 4.1 & 4 & 0 & 0 \\
\hline 4.5 & 4 & 0 & 0 \\
\hline 4.9 & 4 & 0 & 0 \\
\hline 5.3 & 4 & 0 & 0 \\
\hline 5.7 & 4 & 0 & \\
\hline
\end{tabular}

Table 3: Splice detection results for the Blacksville belt. Column labels are as described in table 2. 


\begin{tabular}{|c|c|c|c|}
\hline Threshold & True Positives & False Positives & Missed Detects \\
\hline 2.1 & 15 & 15 & 0 \\
\hline 2.5 & 15 & 6 & 0 \\
\hline 2.9 & 15 & 2 & 0 \\
\hline 3.3 & 15 & 1 & 0 \\
\hline 3.7 & 15 & 1 & 0 \\
\hline 4.1 & 15 & 0 & 0 \\
\hline 4.5 & 15 & 0 & 1 \\
\hline 4.9 & 14 & 0 & 3 \\
\hline 5.3 & 12 & 0 & 3 \\
\hline 5.7 & 12 & 0 & 0 \\
\hline
\end{tabular}

Table 4: Splice detection results for the Blacksville belt. Column labels are as described in table 2.

\begin{tabular}{|c|c|c|c|}
\hline Threshold & True Positives & False Positives & Missed Detects \\
\hline 2.1 & 11 & 127 & 0 \\
\hline 2.3 & 11 & 28 & 0 \\
\hline 2.5 & 11 & 4 & 0 \\
\hline 2.7 & 10 & 0 & 1 \\
\hline 2.9 & 10 & 0 & 1 \\
\hline 3.1 & 10 & 0 & 1 \\
\hline 3.3 & 10 & 0 & 1 \\
\hline 3.5 & 10 & 0 & 1 \\
\hline 3.7 & 10 & 0 & 1 \\
\hline 3.9 & 10 & 0 & 1 \\
\hline
\end{tabular}

Table 5: Splice detection results for the Blacksville belt. Column labels are as described in table 2. 
these rules were implemented, for the next five months (the remaining life in the mining section), the belt did not break. Based on historical data, one would have expected ten belt breaks in the remainder of the longwall panel.

In the time since acquisition of the results, the vulcanized splice detection algorithm has been further modified to eliminate image discontinuities due to camera misalignment. Image data for the BeltVision system comes from two scan-line cameras suspended above the moving belt. Output from the two cameras is concatenated to form a single scan-line of double length. As the belt moves, subsequent scan-lines image different parts of the belt, resulting in a 2D image of the belt surface. By using two cameras, the entire width of the belt can be imaged using inexpensive CCD image sensors, and relatively mild optics. The disadvantage of this approach is that the cameras must be carefully aligned so that the the scan-lines match well. Figures 24(a) and 24(c) show images of vulcanized splices in which camera misalignment gives the impression of a discontinuity in the splice.

The vulcanized splice detection algorithm was modified to include a preprocessing step in which the two halves of the belt image are aligned. The cameras are adjusted so that part of the belt is imaged by both cameras, and the preprocessing algorithm searches for the the 2D translation that most closely aligns the two halves of the image. For the small translations involved, it is not necessary to account for perspective effects.

To perform the alignment, a portion of the image near the center border is assumed to overlap, and image similarity is computed for a range of horizontal and vertical translations. The translation at which the two halves of the image are most similar is assumed to reflect the optimal alignment. As the belt moves, the similarity measures are recursively updated, allowing smooth adjustment to gradual changes in camera position. Figures 24(b) and 24(d) show images of vulcanized splices for which the camera alignment preprocessing step has been performed.

\section{Conclusion}

As stated in the Introduction, the overall objective of the three-year project was to develop and commercialize a low cost conveyor belt inspection system for use in underground and surface mines, coal-fired power generation plants, and other large scale material handling operations. Through DOE funding and cost-share dollars, vision systems have been developed that are both cost effective and accurate. These systems are currently deployed in 16 active mines, and are having daily positive impact on productivity, energy use, and worker safety. A low-cost Smart Camera based version of the system has been developed and is undergoing shake-out testing in preparation for marketing and commercial sales. This has been a successful project, with potential for enormous impact on the

mining industry. 


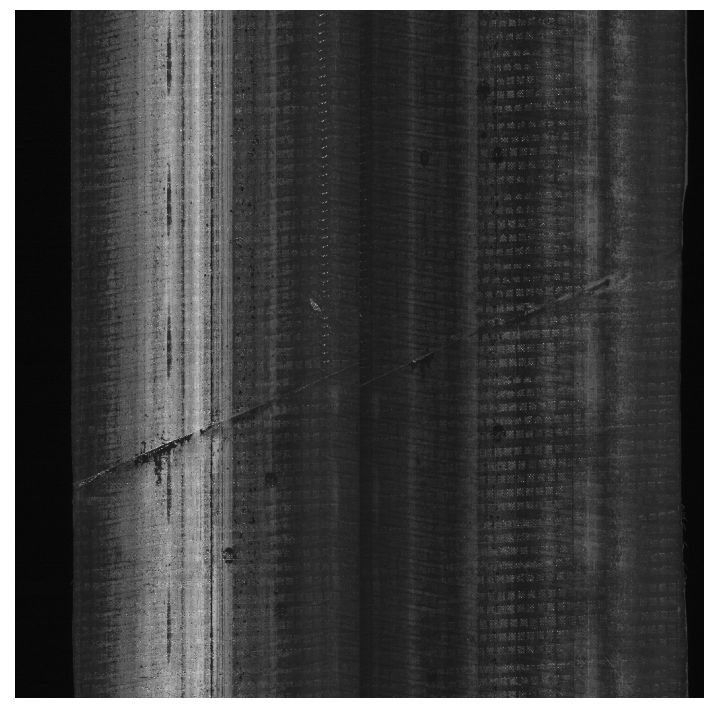

(a)

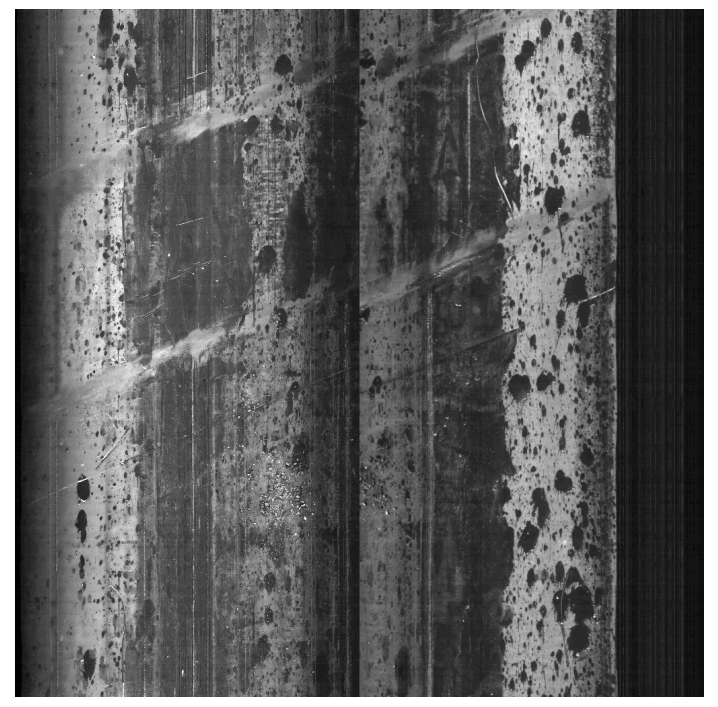

(c)

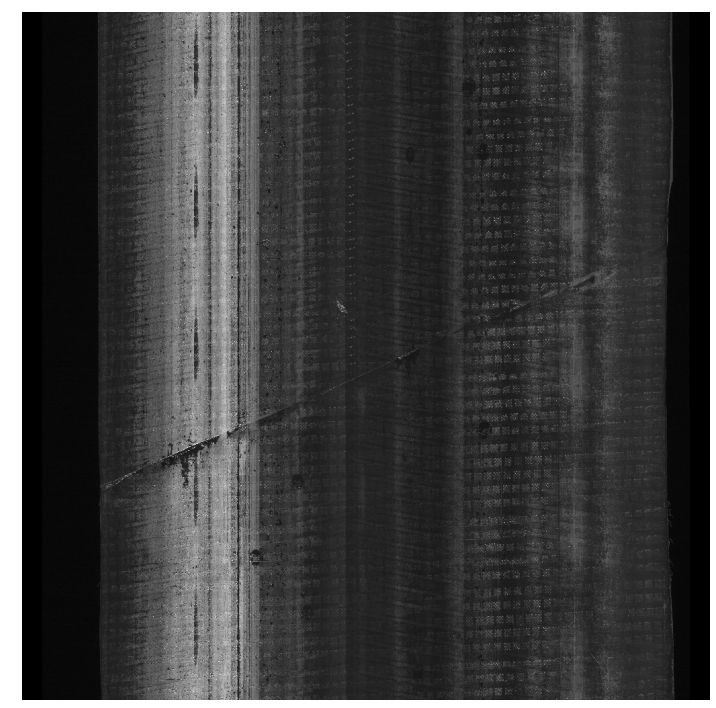

(b)

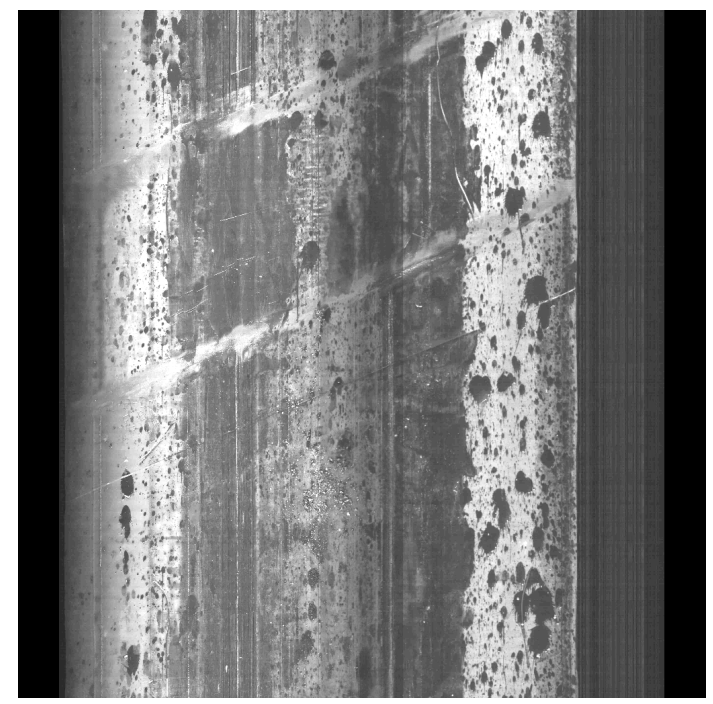

(d)

Figure 24: Belt images showing the effect of the new alignment algorithm. Contrast has been altered to make splice features more visible. (a) An image of a vulcanized splice from the from the Bailey mine without alignment. (b) The same image after alignment. (c) An image of a vulcanized splice from the Robinson Run mine without alignment. (d) The same image after alignment. 